\title{
An Application of Product of Intuitionistic Fuzzy Incidence Graphs in Textile Industry
}

\author{
Irfan Nazeer $\mathbb{D}^{\mathbb{D}},{ }^{1}$ Tabasam Rashid $\mathbb{D}^{\mathbb{D}},{ }^{1}$ and Abazar Keikha $\mathbb{D i D}^{2}$ \\ ${ }^{1}$ University of Management and Technology, Lahore 54770, Pakistan \\ ${ }^{2}$ Velayat University, Iranshahr, Iran \\ Correspondence should be addressed to Abazar Keikha; abazar_keikha@yahoo.com
}

Received 15 January 2021; Revised 4 March 2021; Accepted 29 March 2021; Published 21 April 2021

Academic Editor: Huihua Chen

Copyright (c) 2021 Irfan Nazeer et al. This is an open access article distributed under the Creative Commons Attribution License, which permits unrestricted use, distribution, and reproduction in any medium, provided the original work is properly cited.

In this research article, we presented the idea of intuitionistic fuzzy incidence graphs (IFIGs) along with their certain properties. The number of operations including Cartesian product (CP), composition, tensor product, and normal product in an IFIGs are also investigated. The method to compute the degree of IFIGs obtained by CP, composition, tensor product, and the normal product is discussed. Some important theorems to calculate the degree of the vertices of IFIGs acquired by CP, composition, tensor product, and normal product are elaborated. An application of CP and composition of two IFIGs in the textile industry to find the best combinations of departments expressing the highest percentage of progress and the lowest percentage of nonprogress is provided. A comparative analysis of our study with the existing study is discussed. Our study will be beneficial to comprehend and understand the further characteristics of IFIGs in detail. Another advantage of our study is that it will be helpful to find the maximum percentage of progress and minimum percentage of nonprogress in different departments of universities, garment factories, and hospitals.

\section{Introduction}

Zadeh [1] initiated the idea of a fuzzy set (FS) to resolve difficulties in dealing with uncertainties. Since then, the theory of FSs and fuzzy logic have been examined by many researchers to solve many real-life problems involving an ambiguous and uncertain environment. FS theory becomes a sturdy area in multiple disciplines including mathematics, computer science, and signal processing. FSs were not without flaws, they only talked about the membership function (MSF) and missed the nonmembership function (NMSF). This drawback in FSs leads Attnassov [2] to present the idea of the intuitionistic fuzzy set (IFS) as a generalization of FS that handles unsure situations in a good way as its structure is not restricted to membership (MS) grades only. The concept of IFS is a preferable tool to use due to its sundry structure explaining MS as well as nonmembership (NMS) grades of an element. IFSs have been outstandingly used in different areas of life. De et al. [3] presented some applications of IFSs in medical diagnosis. Xu [4] defined some aggregation operators.
A graph is an easy way of expressing information including an association between entities. The entities are shown by vertices and relations by edges. In different problems, we get incomplete information about the problem. So, there is blurriness in the explanation of the entities or their relationship or both. To tackle this form of problem, we need to design a fuzzy graph (FG) model. Zadeh's FS provided a strong ground for the theory of FGs which have been proposed by Rosenfeld [5]. The study of FGs leads many researchers to contribute in this field; for example, Mordeson and Chang-Shyh [6] explained the various kinds of operations like composition, union, and join on FGs. Pal et al. [7] established the degree and total degree of an edge in interval-valued FGs. Some properties of highly irregular interval-valued FGs were initiated by Rashmanlou and $\mathrm{Pal}$ [8]. Jan et al. [9] discussed some root-level modifications in interval-valued FGs. Mathew et al. [10] introduced vertex rough graphs. Certain types of FGs such as fuzzy threshold graphs, fuzzy tolerance graphs, and Bipolar fuzzy hypergraphs were given by Samanta and Pal [11-13]. For more 
comprehensive and detailed work on graphs and FGs, we may refer to the reader [14-16].

Parvathi and Karunambigai [17] have developed the idea of intuitionistic fuzzy graphs (IFGs). Like IFSs, some outstanding work on the theory of IFGs is also being done. Parvathi et al. [18] discussed different operations on IFGs including CP and composition. Gani and Begum [19] talked about the degree, order, and size of IFGs. Direct product, semistrong product, and strong product in IFGs were discussed by Sahoo and Pal [20]. Sahoo and Pal [21, 22] studied intuitionistic fuzzy competition graphs and intuitionistic fuzzy tolerance graphs with applications. Various kinds of products like Cartesian, tensor, normal, and their degrees in IFGs were provided by Sahoo and Pal [23]. Sahoo et al. [24] initiated new ideas in IFGs with application in the water supply system. Bozhenyuk et al. [25] presented an idea of minimal intuitionistic dominating vertex subset of an IFG.

FGs and IFGs do not talk about the influence of vertices on the edges. This lack in these graphs was caused to introduce an idea of fuzzy incidence graphs (FIGs). For example, if vertices show different residence societies and edges show roads joining these residence societies, we can have a FG expressing the extent of traffic from one society to another. The society that has the maximum number of residents will have maximum ramps in society. So, if $c$ and $\mathrm{d}$ are two societies and $\mathrm{cd}$ is a road joining them then (c, cd) could express the ramp system from the road cd to the society c. In the case of an unweighted graph, both $c$ and $\mathrm{d}$ will have an influence of 1 on $\mathrm{cd}$. In a directed graph, the influence of $c$ on $c d$ represented by $(c, c d)$ is 1 whereas $(d$, cd) is 0 . This concept is generalized through FIGs. Dinesh [26] presented the notion of FIGs. Malik et al. [27, 28] utilized FIGs in human trafficking. The idea of cut-pairs, fuzzy incidence trees, and strong pairs in FIGs was proposed by Mathew and Mordeson [29]. Fang et al. [30] presented an idea to find the connectivity and Wiener index of FIG.

IFGs and different types of operations for IFGs including $\mathrm{CP}$, composition, tensor product (TP), and normal product (NP) exist in literature but these operations are unknown for IFIGs. This motivates us to introduce IFIGs and these operations for IFIGs. IFIGs are more beneficial than FIGs due to the availability of the NMSF in IFIGs. These innovative ideas will open a new door for many researchers to study IFIGs in detail. The remainder of this article is formulated as follows: Section 2 provides some preliminary results which are required to understand the remaining part of the article. The method to find the degree of a vertex in $\mathrm{CP}$ and composition is discussed in Section 3. In Section 4, the idea to find the degree of a vertex in TP and NP is explained. The idea of accurate domination in FIGs is presented in Section 5. A real-life application of $\mathrm{CP}$ and composition in the textile industry is provided in Section 5. A comparative analysis of our study with the existing study is provided in Section 6 . Conclusions and prospects are elaborated in Section 7.

\section{Preliminaries}

A fuzzy subset (FSS) $\mu$ on a set $M$ is a map $\mu: M \longrightarrow[0,1]$. A map $v: M \times M \longrightarrow[0,1]$ is known as a fuzzy relation on $\mu$ if $\nu(a, b) \leq \min \{\mu(a), \mu(b)\}$ for each $a, b \in M$. A $F G$ is a pair $G=(\mu, \nu)$, where $\mu$ is a FSS on a set $V$ and $\nu$ is a fuzzy relation on $\mu$.

Definition 1 (see [23]). An IFS $A$ on the set $X$ is characterized by a mapping $e: X \longrightarrow[0,1]$, which is named as a MF and $f: X \longrightarrow[0,1]$, which is said to be NMF.

Definition 2 (see [23]). An IFG is of the form $G=(V, E, \mu, \nu) \quad$ where $\mu=\left(\mu_{1}, \mu_{2}\right), \quad \nu=\left(\nu_{1}, \nu_{2}\right), \quad$ and $V=\left\{a_{0}, a_{1}, \ldots, a_{n}\right\} \quad$ such that $\mu_{1}: V \longrightarrow[0,1]$ and $\mu_{2}: V \longrightarrow[0,1]$ represent the degree of MS and NMS of the vertex $v_{i} \in V$, respectively, and $0 \leq \mu_{1}+\mu_{2} \leq 1$ for each $a_{i} \in V(i=1,2, \ldots, n)$. Also, $v_{1}: V \times V \longrightarrow[0,1]$ and $v_{2}: V \times V \longrightarrow[0,1] ; v_{1}\left(a_{i}, a_{j}\right)$ and $v_{2}\left(a_{i}, a_{j}\right)$ show the degree of MS and NMS of the edge $\left(a_{i}, a_{j}\right)$, respectively, such that $\nu_{1}\left(a_{i}, a_{j}\right) \leq \wedge\left\{\mu_{1}\left(a_{i}\right), \mu_{1}\left(a_{j}\right)\right\}$ and $\nu_{2}\left(a_{i}, a_{j}\right) \leq \vee\left\{\mu_{2}\left(a_{i}\right)\right.$, $\left.\mu_{2}\left(a_{j}\right)\right\}, 0 \leq v_{1}\left(a_{i}, a_{j}\right)+v_{2}\left(a_{i}, a_{j}\right) \leq 1$ for every $\left(a_{i}, a_{j}\right)$.

Example 1. Here, we include a daily life example of six different countries. As an illustrative case, consider a network (IFG) of six vertices indicating the six different countries. The MS value of the vertices represents the percentage of people who are educated and the NMS value of the vertices indicates the percentage of those people who are uneducated. The MS value of the edges expresses the cooperation of one country with another country to enhance the percentage of educated people and the NMS value denotes the noncooperation with each other.

Figure 1 shows an IFG $G=(V, E, \mu, \nu)$ with $V=(p, q, r, s, u, v)$ and

$$
\begin{gathered}
\mu(p)=(0.5,0.3), \\
\mu(q)=(0.2,0.3), \\
\mu(r)=(0.3,0.1), \\
\mu(s)=(0.4,0.3), \\
\mu(u)=(0.6,0.4), \\
\mu(v)=(0.3,0.5), \\
\nu(p, q)=(0.2,0.3), \\
\nu(p, s)=(0.4,0.3), \\
\nu(p, v)=(0.3,0.5), \\
\nu(q, r)=(0.2,0.3), \\
\nu(q, v)=(0.2,0.5), \\
\nu(r, s)=(0.3,0.3), \\
\nu(r, u)=(0.3,0.4), \\
\nu(s, u)=(0.4,0.4) .
\end{gathered}
$$

In this paper, the minimum operator is expressed by $\wedge$ and the maximum operator is represented by $\mathrm{V}$.

Definition 3 (see [23]). Let $G=(V, E, \mu, \nu)$ be an IFG and $c \in V$; then, its degree is represented by $\mathrm{d}_{G(c)}=\left(\mathrm{d}_{1 G}(c), \mathrm{d}_{2 G}(c)\right)$ and defined by 


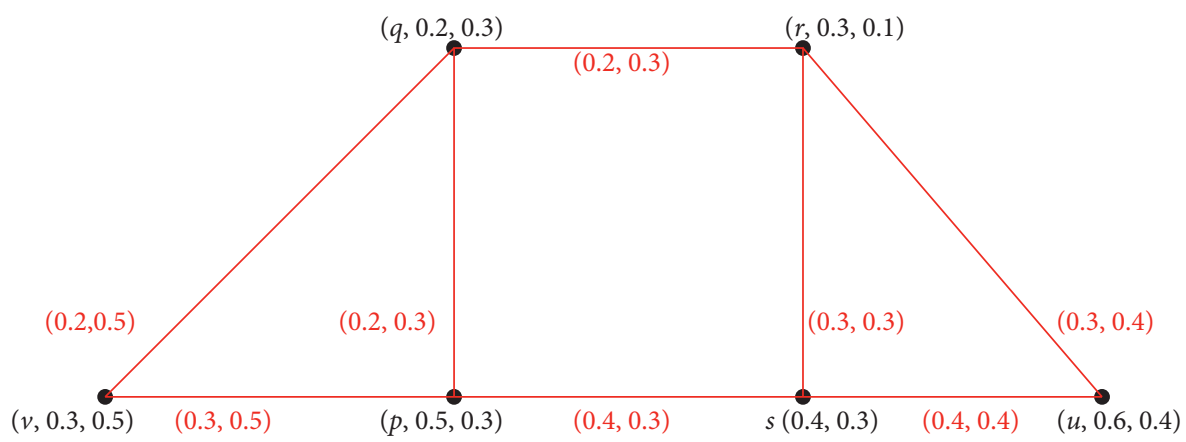

FIgURe 1: An IFG indicating the network of six different countries.

$\mathrm{d}_{1 G}(c)=\sum_{c \neq d} \nu_{1}(c, d)=\sum_{(c, d) \in E} \nu_{1}(c, d) \quad$ and $\quad \mathrm{d}_{2 G}(c)=$ $\sum_{c \neq d} v_{2}(c, d)=\sum_{(c, d) \in E} v_{2}(c, d)$.

Definition 4 (see [26]). Assume $G=(V, E)$ is a graph. Then, $G=(V, E, I)$ is named as an incidence graph (IG), where $I \subseteq V \times E$.

Example 2. An IG is shown in Figure 2. $(a, a b),(b, a b),(b, b c),(c, b c),(a, a c),(c, a c)$ are called incidence pairs (IPs) or pairs of an IG $G=(V, E, I)$.

Definition 5 (see [26]). Assume $G=(V, E)$ is a graph, $\mu$ is a FSS of $\mathrm{V}$, and $\nu$ is a FSS of $V \times V$. Let $\psi$ be a FSS of $V \times E$. If $\psi(a, a b) \leq \mu(a) \wedge \nu(a b)$ for every $a \in V, a b \in E$, then $\psi$ is a fuzzy incidence $(\mathrm{FI})$ of $\mathrm{G}$.

Definition 6 (see [26]). Assume $G$ is a graph and $(\mu, \nu)$ is a fuzzy subgraph of $G$. If $\psi$ is a FI of $G$, then $\widetilde{G}=(\mu, \nu, \psi)$ is named as FIG of $G$.

Example 3. A FIG with $\mu(a)=0.8, \mu(b)=0.3$, $\mu(c)=0.1, \nu(a b)=0.1, \nu(b c)=0.1 \quad$ and $\quad \psi(a, a b)=0.1$, $\psi(b, a b)=0.1, \psi(b, b c)=0.05, \psi(c, b c)=0.07$ is shown in Figure 3.

\section{Degree of a Vertex of CP and Composition of Intuitionistic Fuzzy Incidence Graphs}

In this section, we define the IFIG, CP, and composition of two IFIGs. We also define the degree of a vertex of these products on IFIGs. Theorems are also provided to calculate the degree of a vertex in CP and composition.

Definition 7. An IFIG is of the form $G=(V, E, I, \mu, v, \psi)$ where $\mu=\left(\mu_{1}, \mu_{2}\right), \quad v=\left(\nu_{1}, \nu_{2}\right), \quad \psi=\left(\psi_{1}, \psi_{2}\right)$, and $V=\left\{a_{0}, a_{1}, \ldots, a_{n}\right\} \quad$ such that $\mu_{1}: V \longrightarrow[0,1]$ and $\mu_{2}: V \longrightarrow[0,1]$ represent the degree of MS and NMS of the vertex $a_{i} \in V$, respectively, and $0 \leq \mu_{1}+\mu_{2} \leq 1$ for each $a_{i} \in V(i=1,2, \ldots, n) \quad \nu_{1}: V \times V \longrightarrow[0,1] \quad$ and $v_{2}: V \times V \longrightarrow[0,1] ; \nu_{1}\left(a_{i}, a_{j}\right)$ and $v_{2}\left(a_{i}, a_{j}\right)$ show the degree of MS and NMS of the edge $\left(a_{i}, a_{j}\right)$, respectively, such that $\nu_{1}\left(a_{i}, a_{j}\right) \leq \wedge\left\{\mu_{1}\left(a_{i}\right), \mu_{1}\left(a_{j}\right)\right\} \quad$ and $\nu_{2}\left(a_{i}, a_{j}\right) \leq \mathrm{V}$ $\left\{\mu_{2}\left(a_{i}\right), \mu_{2}\left(a_{j}\right)\right\}, \quad 0 \leq v_{1}\left(a_{i}, a_{j}\right)+v_{2}\left(a_{i}, a_{j}\right) \leq 1$ for every $\left(a_{i}, a_{j}\right) \quad \psi_{1}: V \times E \longrightarrow[0,1]$ and $\psi_{2}: V \times E \longrightarrow[0,1]$;

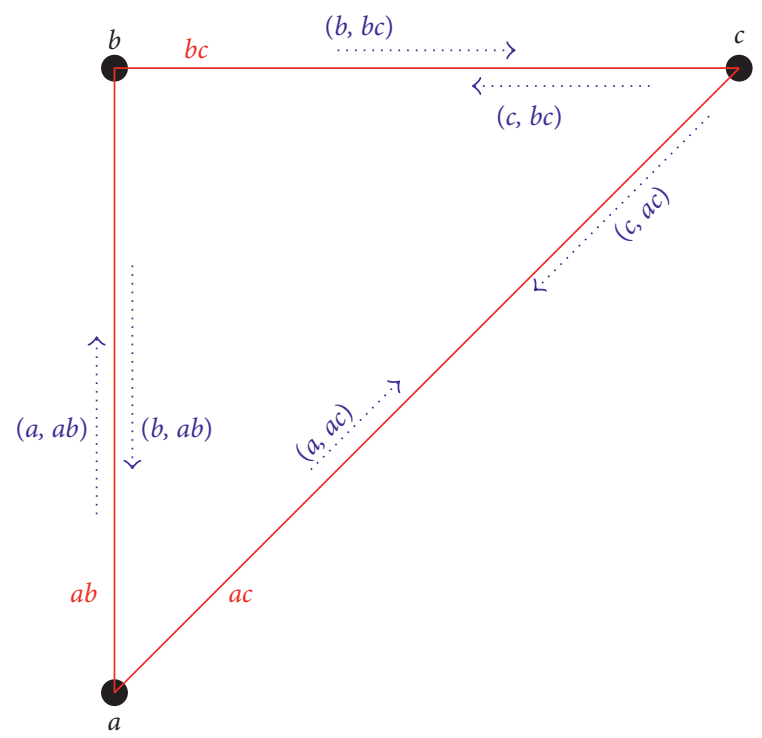

FIgURE 2: Incidence graph.

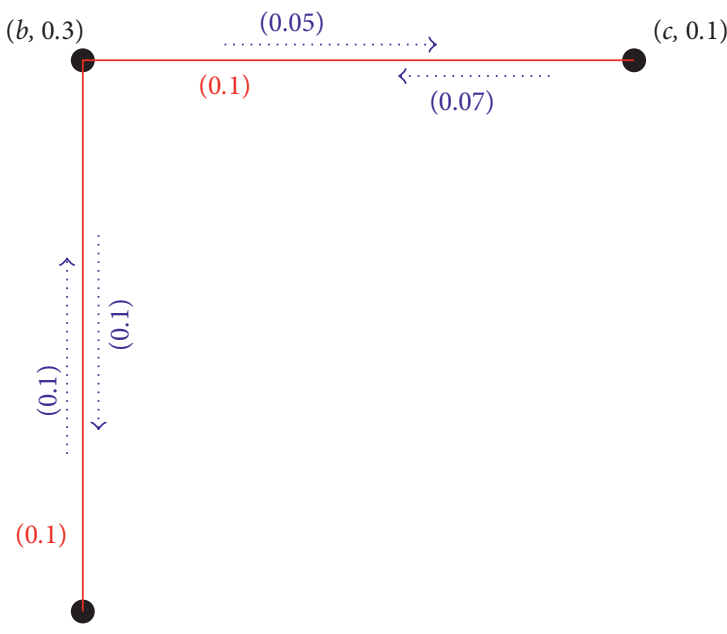

$(a, 0.8)$

Figure 3: A figure.

$\psi_{1}\left(a_{i}, a_{i} a_{j}\right)$ and $\psi_{2}\left(a_{i}, a_{i} a_{j}\right)$ show the degree of MS and NMS of the incidence pair, respectively, such that $\psi_{1}\left(a_{i}, a_{i} a_{j}\right) \leq \wedge\left\{\mu_{1}\left(a_{i}\right), v_{1}\left(a_{i} a_{j}\right)\right\}$ and $\psi_{2}\left(a_{i}, a_{i} a_{j}\right) \leq \wedge\left\{\mu_{2}\right.$ 
$\left.\left(a_{i}\right), v_{2}\left(a_{i} a_{j}\right)\right\}, 0 \leq \psi_{1}\left(a_{i}, a_{i} a_{j}\right)+\psi_{2}\left(a_{i}, a_{i} a_{j}\right) \leq 1$ for every $\left(a_{i}, a_{i} a_{j}\right)$.

Example 4. Here, we include a daily life example of three different shopping malls. As an illustrative case, consider a network (IFIG) of three vertices indicating the three different branches of a shopping mall. The MS value of the vertices represents the number of people who visit the shopping mall for shopping and the NMS value of the vertices indicates those people who do not come to these shopping malls for shopping. The MS value of the edges expresses their cooperation with each other and the NMS value denotes the noncooperation with each other. The MS value of the IPs shows the percentage of profit and the NMS value indicates the percentage of nonprofit of these shopping malls.

Let $G=(V, E, I, \mu, \nu, \psi)$ be an IFIG provided in Figure 4, expressing a network of three branches of a shopping mall where $\mu(a)=\left(\mu_{1}(a), \mu_{2}(a)\right), \nu(a, b)=\left(\nu_{1}(a, b), v_{2}(a, b)\right)$, and $\psi(a, a b)=\left(\psi_{1}(a, a b), \psi_{2}(a, a b)\right)$. Let $V=(a, b, c)$ and $\mu(a)=(0.2,0.6), \mu(b)=(0.1,0.1), \mu(c)=(0.3,0.1)$, $\nu(a, b)=(0.1,0.1), v(b, c)=(0.1,0.1), \quad v(a, c)=(0.2,0.1)$, and $\psi(a, a b)=(0.1,0.1), \psi(b, a b)=(0.1,0.1), \quad \psi(b, b c)=$ $(0.1,0.1), \psi(c, b c)=(0.1,0.05), \psi(a, a c)=(0.1,0.07), \psi \quad(c$, ac) $=(0.1,0.04)$.

Definition 8. Consider $G=(V, E, I, \mu, \nu, \psi)$ is an IFIG and $a \in V$; then, its degree is expressed by $\mathrm{d}_{G(a)}=\left(\mathrm{d}_{1 G}\right.$ $(a), \mathrm{d}_{2 G}(a)$ ) and defined by $\mathrm{d}_{1 G}(a)=\Sigma_{a \neq b,(a, a b) \in I}$ and $\mathrm{d}_{2 G}(a)=\sum_{a \neq b,(a, a b) \in I}$.

Now, we are going to define the CP of two IFIGs and how to find the degree of these graphs.

Definition 9. The CP of two IFIGs $G^{i}=\left(V^{i}, E^{i}, I^{i}, \mu^{i}, \nu^{i}, \psi^{i}\right)$ and $G^{i i}=\left(V^{i i}, E^{i i}, I^{i i}, \mu^{i i}, \nu^{i i}, \psi^{i i}\right)$ is defined as an IFIG $G=$ $G^{i} \times G^{i i}=\left(V, E, I, \mu^{i} \times \mu^{i i}, \nu^{i} \times \nu^{i i}, \psi^{i} \times \psi^{i i}\right)$ where $V=V^{i} \times$ $V^{i i}, E=\left\{\left(\left(x_{1}, y_{1}\right),\left(x_{2}, y_{2}\right)\right) \mid x_{1}=x_{2},\left(y_{1}, y_{2}\right) \in E^{i i}\right.$ or $y_{1}=$ $\left.y_{2},\left(x_{1}, x_{2}\right) \in E^{i}\right\}, \quad$ and $I=\left\{\left(x_{1}, y_{1}\right),\left(x_{1}, y_{1}\right)\left(x_{1}, \quad y_{2}\right)\right.$ $\mid x_{1}=x_{2},\left(y_{1}, y_{1} y_{2}\right) \in I^{i i},\left(y_{2}, y_{1} y_{2}\right) \in I^{i i}$ or $y_{1}=y_{2}, \quad\left(x_{1}\right.$, $\left.\left.x_{1} x_{2}\right) \in I^{i},\left(x_{2}, x_{1} x_{2}\right) \in I^{i}\right\} \quad$ with $\left(\mu_{1}^{i} \times \mu_{1}^{i i}\right)\left(x_{1}, y_{1}\right)=\mu_{1}^{i}$ $\left(x_{1}\right) \wedge \mu_{1}^{i i}\left(y_{1}\right), \forall\left(x_{1}, y_{1}\right) \in V^{i} \times V^{i i}, \quad\left(\mu_{2}^{i} \times \mu_{2}^{i i}\right)\left(x_{1}, y_{1}\right)=\mu_{2}^{i}$ $\left(x_{1}\right) \vee \mu_{2}^{i i}\left(y_{1}\right), \forall\left(x_{1}, y_{1}\right) \in V^{i} \times V^{i i}$.

$$
\begin{aligned}
& \left(v_{1}^{i} \times v_{1}^{i i}\right)\left(\left(x_{1}, y_{1}\right)\left(x_{2}, y_{2}\right)\right)= \begin{cases}\mu_{1}^{i}\left(x_{1}\right) \wedge v_{1}^{i i}\left(y_{1}, y_{2}\right), & \text { if } x_{1}=x_{2},\left(y_{1}, y_{2}\right) \in E^{i i}, \\
v_{1}^{i}\left(x_{1}, x_{2}\right) \wedge \mu_{1}^{i i}\left(y_{1}\right), & \text { if } y_{1}=y_{2},\left(x_{1}, x_{2}\right) \in E^{i},\end{cases} \\
& \left(v_{2}^{i} \times v_{2}^{i i}\right)\left(\left(x_{1}, y_{1}\right)\left(x_{2}, y_{2}\right)\right)= \begin{cases}\mu_{2}^{i}\left(x_{1}\right) \vee v_{2}^{i i}\left(y_{1}, y_{2}\right), & \text { if } x_{1}=x_{2},\left(y_{1}, y_{2}\right) \in E^{i i}, \\
v_{2}^{i}\left(x_{1}, x_{2}\right) \vee \mu_{2}^{i i}\left(y_{1}\right), & \text { if } y_{1}=y_{2},\left(x_{1}, x_{2}\right) \in E^{i},\end{cases} \\
& \left(\psi_{1}^{i} \times \psi_{1}^{i i}\right)\left(\left(x_{1}, y_{1}\right),\left(x_{1}, y_{1}\right)\left(x_{1}, y_{2}\right)\right)=\mu_{1}^{i}\left(x_{1}\right) \wedge \psi_{1}^{i i}\left(y_{1}, y_{1} y_{2}\right), \quad \text { if } x_{1}=x_{2},\left(y_{1}, y_{1} y_{2}\right) \in I^{i i} \text {, } \\
& \left(\psi_{1}^{i} \times \psi_{1}^{i i}\right)\left(\left(x_{1}, y_{2}\right),\left(x_{1}, y_{1}\right)\left(x_{1}, y_{2}\right)\right)=\mu_{1}^{i}\left(x_{1}\right) \wedge \psi_{1}^{i i}\left(y_{2}, y_{1} y_{2}\right), \quad \text { if } x_{1}=x_{2},\left(y_{2}, y_{1} y_{2}\right) \in I^{i i} \text {, } \\
& \left(\psi_{1}^{i} \times \psi_{1}^{i i}\right)\left(\left(x_{1}, y_{1}\right),\left(x_{1}, y_{1}\right)\left(x_{2}, y_{1}\right)\right)=\psi_{1}^{i}\left(x_{1}, x_{1} x_{2}\right) \wedge \mu_{1}^{i i}\left(y_{1}\right), \quad \text { if } y_{1}=y_{2},\left(x_{1}, x_{1} x_{2}\right) \in I^{i}, \\
& \left(\psi_{1}^{i} \times \psi_{1}^{i i}\right)\left(\left(x_{2}, y_{1}\right),\left(x_{1}, y_{1}\right)\left(x_{2}, y_{1}\right)\right)=\psi_{1}^{i}\left(x_{2}, x_{1} x_{2}\right) \wedge \mu_{1}^{i i}\left(y_{1}\right), \quad \text { if } y_{1}=y_{2},\left(x_{2}, x_{1} x_{2}\right) \in I^{i} \text {, } \\
& \left(\psi_{1}^{i} \times \psi_{1}^{i i}\right)\left(\left(x_{1}, y_{2}\right),\left(x_{1}, y_{2}\right)\left(x_{2}, y_{2}\right)\right)=\psi_{1}^{i}\left(x_{1}, x_{1} x_{2}\right) \wedge \mu_{1}^{i i}\left(y_{2}\right), \quad \text { if } y_{1}=y_{2},\left(x_{1}, x_{1} x_{2}\right) \in I^{i} \text {, } \\
& \left(\psi_{1}^{i} \times \psi_{1}^{i i}\right)\left(\left(x_{2}, y_{2}\right),\left(x_{1}, y_{2}\right)\left(x_{2}, y_{2}\right)\right)=\psi_{1}^{i}\left(x_{2}, x_{1} x_{2}\right) \wedge \mu_{1}^{i i}\left(y_{2}\right), \quad \text { if } y_{1}=y_{2},\left(x_{2}, x_{1} x_{2}\right) \in I^{i} \text {, } \\
& \left(\psi_{1}^{i} \times \psi_{1}^{i i}\right)\left(\left(x_{2}, y_{1}\right),\left(x_{2}, y_{1}\right)\left(x_{2}, y_{2}\right)\right)=\psi_{1}^{i i}\left(y_{1}, y_{1} y_{2}\right) \wedge \mu_{1}^{i}\left(x_{2}\right), \quad \text { if } x_{1}=x_{2},\left(y_{1}, y_{1} y_{2}\right) \in I^{i i} \text {, } \\
& \left(\psi_{1}^{i} \times \psi_{1}^{i i}\right)\left(\left(x_{2}, y_{2}\right),\left(x_{2}, y_{1}\right)\left(x_{2}, y_{2}\right)\right)=\psi_{1}^{i i}\left(y_{2}, y_{1} y_{2}\right) \wedge \mu_{1}^{i}\left(x_{2}\right), \quad \text { if } x_{1}=x_{2},\left(y_{2}, y_{1} y_{2}\right) \in I^{i i} \text {, } \\
& \left(\psi_{2}^{i} \times \psi_{2}^{i i}\right)\left(\left(x_{1}, y_{1}\right),\left(x_{1}, y_{1}\right)\left(x_{1}, y_{2}\right)\right)=\mu_{2}^{i}\left(x_{1}\right) \vee \psi_{2}^{i i}\left(y_{1}, y_{1} y_{2}\right), \quad \text { if } x_{1}=x_{2},\left(y_{1}, y_{1} y_{2}\right) \in I^{i i} \text {, } \\
& \left(\psi_{2}^{i} \times \psi_{2}^{i i}\right)\left(\left(x_{1}, y_{2}\right),\left(x_{1}, y_{1}\right)\left(x_{1}, y_{2}\right)\right)=\mu_{2}^{i}\left(x_{1}\right) \vee \psi_{2}^{i i}\left(y_{2}, y_{1} y_{2}\right), \quad \text { if } x_{1}=x_{2},\left(y_{2}, y_{1} y_{2}\right) \in I^{i i} \text {, } \\
& \left(\psi_{2}^{i} \times \psi_{2}^{i i}\right)\left(\left(x_{1}, y_{1}\right),\left(x_{1}, y_{1}\right)\left(x_{2}, y_{1}\right)\right)=\psi_{2}^{i}\left(x_{1}, x_{1} x_{2}\right) \vee \mu_{2}^{i i}\left(y_{1}\right), \quad \text { if } y_{1}=y_{2},\left(x_{1}, x_{1} x_{2}\right) \in I^{i} \text {, } \\
& \left(\psi_{2}^{i} \times \psi_{2}^{i i}\right)\left(\left(x_{2}, y_{1}\right),\left(x_{1}, y_{1}\right)\left(x_{2}, y_{1}\right)\right)=\psi_{2}^{i}\left(x_{2}, x_{1} x_{2}\right) \vee \mu_{2}^{i i}\left(y_{1}\right), \quad \text { if } y_{1}=y_{2},\left(x_{2}, x_{1} x_{2}\right) \in I^{i} \text {, } \\
& \left(\psi_{2}^{i} \times \psi_{2}^{i i}\right)\left(\left(x_{1}, y_{2}\right),\left(x_{1}, y_{2}\right)\left(x_{2}, y_{2}\right)\right)=\psi_{2}^{i}\left(x_{1}, x_{1} x_{2}\right) \vee \mu_{2}^{i i}\left(y_{2}\right), \quad \text { if } y_{1}=y_{2},\left(x_{1}, x_{1} x_{2}\right) \in I^{i} \text {, } \\
& \left(\psi_{2}^{i} \times \psi_{2}^{i i}\right)\left(\left(x_{2}, y_{2}\right),\left(x_{1}, y_{2}\right)\left(x_{2}, y_{2}\right)\right)=\psi_{2}^{i}\left(x_{2}, x_{1} x_{2}\right) \vee \mu_{2}^{i i}\left(y_{2}\right), \quad \text { if } y_{1}=y_{2},\left(x_{2}, x_{1} x_{2}\right) \in I^{i}, \\
& \left(\psi_{2}^{i} \times \psi_{2}^{i i}\right)\left(\left(x_{2}, y_{1}\right),\left(x_{2}, y_{1}\right)\left(x_{2}, y_{2}\right)\right)=\psi_{2}^{i i}\left(y_{1}, y_{1} y_{2}\right) \vee \mu_{2}^{i}\left(x_{2}\right), \quad \text { if } x_{1}=x_{2},\left(y_{1}, y_{1} y_{2}\right) \in I^{i i} \text {, } \\
& \left(\psi_{2}^{i} \times \psi_{2}^{i i}\right)\left(\left(x_{2}, y_{2}\right),\left(x_{2}, y_{1}\right)\left(x_{2}, y_{2}\right)\right)=\psi_{2}^{i i}\left(y_{2}, y_{1} y_{2}\right) \vee \mu_{2}^{i}\left(x_{2}\right), \quad \text { if } x_{1}=x_{2},\left(y_{2}, y_{1} y_{2}\right) \in I^{i i} \text {. }
\end{aligned}
$$




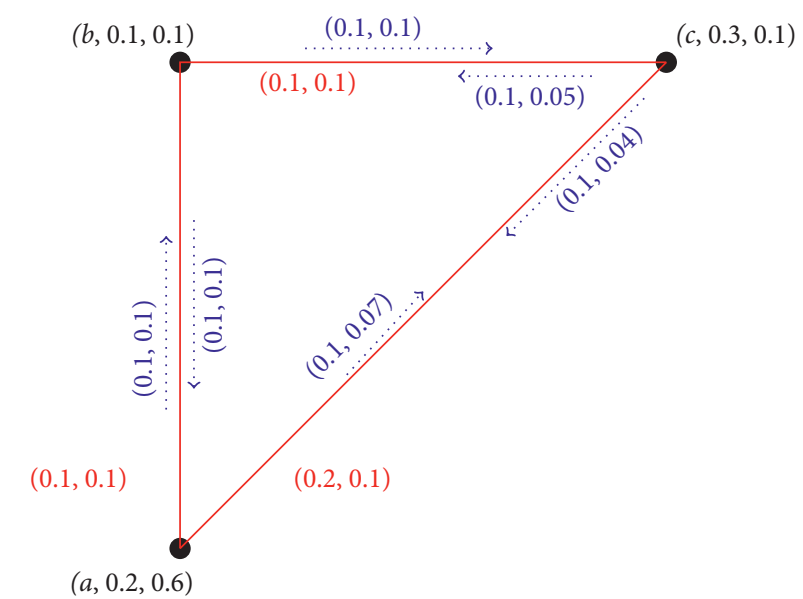

Figure 4: A network of different branches of the shopping mall.

Definition 10. Let $G=G^{i} \times G^{i i}=\left(V, E, I, \mu^{i} \quad \times \mu^{i i}, \nu^{i}\right.$ $\left.\times v^{i i}, \psi^{i} \times \psi^{i i}\right)$ be the CP of two IFIGs $G^{i}=\left(V^{i}, E^{i}, I^{i}, \mu^{i}, \nu^{i}, \psi^{i}\right)$ and $G^{i i}=\left(V^{i i}, E^{i i}, I^{i i}, \mu^{i i}, \nu^{i i}, \psi^{i i}\right)$. Then, the degree of $V=$ $\left(x_{1}, y_{1}\right)$ is expressed by $\mathrm{d}_{G^{i} \times G^{i i}}\left(x_{1}, y_{1}\right)=$ $\left(\mathrm{d}_{1 G^{i} \times G^{i i}}\left(x_{1}, y_{1}\right), \mathrm{d}_{2 G^{i} \times G^{i i}}\left(x_{1}, y_{1}\right)\right)$ and defined by

$$
\begin{aligned}
& \mathrm{d}_{1 G^{i} \times G^{i i}}\left(x_{1}, y_{1}\right)=\sum_{x_{1}=x_{2},\left(y_{1}, y_{1} y_{2}\right) \in I^{i i}} \mu_{1}^{i}\left(x_{1}\right) \wedge \psi_{1}^{i i}\left(y_{1}, y_{1} y_{2}\right)+\sum_{y_{1}=y_{2},\left(x_{1}, x_{1} x_{2}\right) \in I^{i}} \psi_{1}^{i}\left(x_{1}, x_{1} x_{2}\right) \wedge \mu_{1}^{i i}\left(y_{1}\right), \\
& \mathrm{d}_{2 G^{i} \times G^{i i}}\left(x_{1}, y_{1}\right)=\sum_{x_{1}=x_{2},\left(y_{1}, y_{1} y_{2}\right) \in I^{i i}} \mu_{2}^{i}\left(x_{1}\right) \vee \psi_{2}^{i i}\left(y_{1}, y_{1} y_{2}\right)+\sum_{y_{1}=y_{2},\left(x_{1}, x_{1} x_{2}\right) \in I^{i}} \psi_{2}^{i}\left(x_{1}, x_{1} x_{2}\right) \vee \mu_{2}^{i i}\left(y_{1}\right) .
\end{aligned}
$$

Theorem 1. Let $G^{i}=\left(V^{i}, E^{i}, I^{i}, \mu^{i}, \nu^{i}, \psi^{i}\right) \quad$ and $G^{i i}=\left(V^{i i}, E^{i i}, I^{i i}, \mu^{i i}, \nu^{i i}, \psi^{i i}\right)$ be two IFIGs. If $\mu_{1}^{i} \geq \psi_{1}^{i i}, \mu_{2}^{i} \leq \psi_{2}^{i i}$ and $\mu_{1}^{i i} \geq \psi_{1}^{i}, \mu_{2}^{i i} \leq \psi_{2}^{i}$, then $d_{G^{i} \times G^{i i}}\left(x_{1}, y_{1}\right)=d_{G^{i}}\left(x_{1}\right)$ $+d_{G^{i i}}\left(y_{1}\right)$.

Proof. In CP, by the definition of the degree of a vertex, we have

$$
\begin{aligned}
\mathrm{d}_{1 G^{i} \times G^{i i}}\left(x_{1}, y_{1}\right) & =\sum_{x_{1}=x_{2},\left(y_{1}, y_{1} y_{2}\right) \in I^{i i}} \mu_{1}^{i}\left(x_{1}\right) \wedge \psi_{1}^{i i}\left(y_{1}, y_{1} y_{2}\right)+\sum_{y_{1}=y_{2},\left(x_{1}, x_{1} x_{2}\right) \in I^{i}} \psi_{1}^{i}\left(x_{1}, x_{1} x_{2}\right) \wedge \mu_{1}^{i i}\left(y_{1}\right), \\
& =\sum_{\left(y_{1}, y_{1} y_{2}\right) \in I^{i i}} \psi_{1}^{i i}\left(y_{1}, y_{1} y_{2}\right)+\sum_{\left(x_{1}, x_{1} x_{2}\right) \in I^{i}} \psi_{1}^{i}\left(x_{1}, x_{1} x_{2}\right) .
\end{aligned}
$$

Since $\mu_{1}^{i} \geq \psi_{1}^{i i}$ and $\mu_{1}^{i i} \geq \psi_{1}^{i}$,

$$
\begin{aligned}
& =\sum_{\left(x_{1}, x_{1} x_{2}\right) \in I^{i}} \psi_{1}^{i}\left(x_{1}, x_{1} x_{2}\right)+\sum_{\left(y_{1}, y_{1} y_{2}\right) \in I^{i i}} \psi_{1}^{i i}\left(y_{1}, y_{1} y_{2}\right) \\
& =\mathrm{d}_{1 G^{i}}\left(x_{1}\right)+\mathrm{d}_{1 G^{i i}}\left(y_{1}\right), \\
\mathrm{d}_{2 G^{i} \times G^{i i}}\left(x_{1}, y_{1}\right) & =\sum_{x_{1}=x_{2},\left(y_{1}, y_{1} y_{2}\right) \in I^{i i}} \mu_{2}^{i}\left(x_{1}\right) \vee \psi_{2}^{i i}\left(y_{1}, y_{1} y_{2}\right)+\sum_{y_{1}=y_{2},\left(x_{1}, x_{1} x_{2}\right) \in I^{i}} \psi_{2}^{i}\left(x_{1}, x_{1} x_{2}\right) \vee \mu_{2}^{i i}\left(y_{1}\right) \\
& =\sum_{\left(y_{1}, y_{1} y_{2}\right) \in I^{i i}} \psi_{2}^{i i}\left(y_{1}, y_{1} y_{2}\right)+\sum_{\left(x_{1}, x_{1} x_{2}\right) \in I^{i}} \psi_{2}^{i}\left(x_{1}, x_{1} x_{2}\right) .
\end{aligned}
$$

Since $\mu_{2}^{i} \leq \psi_{2}^{i i}$ and $\mu_{2}^{i i} \leq \psi_{2}^{i}$, 


$$
\begin{aligned}
& =\sum_{\left(x_{1}, x_{1} x_{2}\right) \in I^{i}} \psi_{2}^{i}\left(x_{1}, x_{1} x_{2}\right)+\sum_{\left(y_{1}, y_{1} y_{2}\right) \in I^{i i}} \psi_{2}^{i i}\left(y_{1}, y_{1} y_{2}\right) \\
& =\mathrm{d}_{2 G^{i}}\left(x_{1}\right)+\mathrm{d}_{2 G^{i i}}\left(y_{1}\right) .
\end{aligned}
$$

Hence, $\mathrm{d}_{G^{i} \times G^{i i}}\left(x_{1}, y_{1}\right)=\mathrm{d}_{G^{i}}\left(x_{1}\right)+\mathrm{d}_{G^{i i}}\left(y_{1}\right)$.

Example 5. In Figures 5 and $6, \mu_{1}^{i} \geq \psi_{1}^{i i}, \mu_{2}^{i} \leq \psi_{2}^{i i}$, and $\mu_{1}^{i i} \geq \psi_{1}^{i}, \mu_{2}^{i i} \leq \psi_{2}^{i}$. Then, by Theorem 1 , we have

$$
\begin{aligned}
& \mathrm{d}_{1 G^{i} \times G^{i i}}\left(x_{1}, y_{1}\right)=\mathrm{d}_{1 G^{i}}\left(x_{1}\right)+\mathrm{d}_{1 G^{i i}}\left(y_{1}\right)=0.2+0.1=0.3, \\
& \mathrm{~d}_{2 G^{i} \times G^{i i}}\left(x_{1}, y_{1}\right)=\mathrm{d}_{2 G^{i}}\left(x_{1}\right)+\mathrm{d}_{2 G^{i i}}\left(y_{1}\right)=0.4+0.4=0.8 .
\end{aligned}
$$

So, $\mathrm{d}_{G^{i} \times G^{i i}}\left(x_{1}, y_{1}\right)=(0.3,0.8)$. In a similar manner, we can examine the degree of all the vertices of $\mathrm{CP}$ of Figures 5 and 6 provided in Figure 7.
Next, the method to explore the degree of a vertex in the composition of two IFIGs is provided.

Definition 11. The composition of two IFIGs $G^{i}=\left(V^{i}, E^{i}, I^{i}, \mu^{i}, \nu^{i}, \psi^{i}\right)$ and $G^{i i}=\left(V^{i i}, E^{i i}, I^{i i}, \mu^{i i}, \nu^{i i}, \psi^{i i}\right)$ is defined as an IFIG $G=G^{i}\left[G^{i i}\right]=\left(V, E, I, \mu^{i} \odot\right.$ $\left.\mu^{i i}, \nu^{i} \odot \nu^{i i}, \psi^{i} \odot \psi^{i i}\right)$ where $V=V^{i} \times V^{i i}, \quad E=\left\{\left(\left(x_{1}, y_{1}\right)\right.\right.$, $\left.\left(x_{2}, y_{2}\right)\right) \mid x_{1}=x_{2},\left(y_{1}, y_{2}\right) \in E^{i i}$ or $\left.y_{1}=y_{2},\left(x_{1}, x_{2}\right) \in E^{i}\right\}$ $\cup\left\{\left(\left(x_{1}, y_{1}\right),\left(x_{2}, y_{2}\right)\right) \mid y_{1} \neq y_{2},\left(x_{1}, x_{2}\right) \in E^{i}\right\}$, and $I=\left\{\left(\left(x_{1}\right.\right.\right.$, $\left.\left.y_{1}\right),\left(x_{1}, y_{1}\right)\left(x_{1}, y_{2}\right)\right) \mid x_{1}=x_{2},\left(y_{1}, y_{1} y_{2}\right) \in I^{i i}, \quad\left(y_{2}, y_{1} y_{2}\right)$ $\in I^{i i} \quad$ or $\left.\quad y_{1}=y_{2},\left(x_{1}, x_{1} x_{2}\right) \quad \in I^{i},\left(x_{2}, x_{1} x_{2}\right) \in I^{i}\right\}$ $\cup\left\{\left(x_{1}, y_{1}\right),\left(x_{2}, y_{2}\right) \mid y_{1} \neq y_{2},\left(x_{1}, x_{1} x_{2}\right) \in I^{i},\left(x_{2}, x_{1} x_{2}\right) \in I^{i}\right\}$ with

$$
\begin{aligned}
& \left(\mu_{1}^{i} \odot \mu_{1}^{i i}\right)\left(x_{1}, y_{1}\right)=\mu_{1}^{i}\left(x_{1}\right) \wedge \mu_{1}^{i i}\left(y_{1}\right), \forall\left(x_{1}, y_{1}\right) \in V^{i} \odot V^{i i} \text {, } \\
& \left(\mu_{2}^{i} \odot \mu_{2}^{i i}\right)\left(x_{1}, y_{1}\right)=\mu_{2}^{i}\left(x_{1}\right) \vee \mu_{2}^{i i}\left(y_{1}\right), \forall\left(x_{1}, y_{1}\right) \in V^{i} \odot V^{i i} \text {, } \\
& \left(\nu_{1}^{i} \odot \nu_{1}^{i i}\right)\left(\left(x_{1}, y_{1}\right)\left(x_{2}, y_{2}\right)\right)= \begin{cases}\mu_{1}^{i}\left(x_{1}\right) \wedge v_{1}^{i i}\left(y_{1}, y_{2}\right), & \text { if } x_{1}=x_{2},\left(y_{1}, y_{2}\right) \in E^{i i}, \\
\nu_{1}^{i}\left(x_{1}, x_{2}\right) \wedge \mu_{1}^{i i}\left(y_{1}\right), & \text { if } y_{1}=y_{2},\left(x_{1}, x_{2}\right) \in E^{i}, \\
v_{1}^{i}\left(x_{1}, x_{2}\right) \wedge \mu_{1}^{i i}\left(y_{1}\right) \wedge \mu_{1}^{i i}\left(y_{2}\right), & \text { if } y_{1} \neq y_{2},\left(x_{1}, x_{2}\right) \in E^{i},\end{cases} \\
& \left(v_{2}^{i} \odot \nu_{2}^{i i}\right)\left(\left(x_{1}, y_{1}\right)\left(x_{2}, y_{2}\right)\right)= \begin{cases}\mu_{2}^{i}\left(x_{1}\right) \vee v_{2}^{i i}\left(y_{1}, y_{2}\right), & \text { if } x_{1}=x_{2},\left(y_{1}, y_{2}\right) \in E^{i i}, \\
v_{2}^{i}\left(x_{1}, x_{2}\right) \vee \mu_{2}^{i i}\left(y_{1}\right), & \text { if } y_{1}=y_{2},\left(x_{1}, x_{2}\right) \in E^{i}, \\
v_{1}^{i}\left(x_{1}, x_{2}\right) \vee \mu_{1}^{i i}\left(y_{1}\right) \vee \mu_{1}^{i i}\left(y_{2}\right), & \text { if } y_{1} \neq y_{2},\left(x_{1}, x_{2}\right) \in E^{i},\end{cases}
\end{aligned}
$$

$\left(\psi_{1}^{i} \odot \psi_{1}^{i i}\right)\left(\left(x_{1}, y_{1}\right),\left(x_{1}, y_{1}\right)\left(x_{1}, y_{2}\right)\right)=\mu_{1}^{i}\left(x_{1}\right) \wedge \psi_{1}^{i i}\left(y_{1}, y_{1} y_{2}\right), \quad$ if $x_{1}=x_{2},\left(y_{1}, y_{1} y_{2}\right) \in I^{i i}$,

$\left(\psi_{1}^{i} \odot \psi_{1}^{i i}\right)\left(\left(x_{1}, y_{2}\right),\left(x_{1}, y_{1}\right)\left(x_{1}, y_{2}\right)\right)=\mu_{1}^{i}\left(x_{1}\right) \wedge \psi_{1}^{i i}\left(y_{2}, y_{1} y_{2}\right), \quad$ if $x_{1}=x_{2},\left(y_{2}, y_{1} y_{2}\right) \in I^{i i}$,

$\left(\psi_{1}^{i} \odot \psi_{1}^{i i}\right)\left(\left(x_{1}, y_{1}\right),\left(x_{1}, y_{1}\right)\left(x_{2}, y_{1}\right)\right)=\psi_{1}^{i}\left(x_{1}, x_{1} x_{2}\right) \wedge \mu_{1}^{i i}\left(y_{1}\right), \quad$ if $y_{1}=y_{2},\left(x_{1}, x_{1} x_{2}\right) \in I^{i}$,

$\left(\psi_{1}^{i} \odot \psi_{1}^{i i}\right)\left(\left(x_{2}, y_{1}\right),\left(x_{1}, y_{1}\right)\left(x_{2}, y_{1}\right)\right)=\psi_{1}^{i}\left(x_{2}, x_{1} x_{2}\right) \wedge \mu_{1}^{i i}\left(y_{1}\right), \quad$ if $y_{1}=y_{2},\left(x_{2}, x_{1} x_{2}\right) \in I^{i}$,

$\left(\psi_{1}^{i} \odot \psi_{1}^{i i}\right)\left(\left(x_{1}, y_{1}\right),\left(x_{1}, y_{1}\right)\left(x_{2}, y_{2}\right)\right)=\psi_{1}^{i}\left(x_{1}, x_{1} x_{2}\right) \wedge \mu_{1}^{i i}\left(y_{1}\right) \wedge \mu_{1}^{i i}\left(y_{2}\right), \quad$ if $x_{1} \neq x_{2}, y_{1} \neq y_{2},\left(x_{1}, x_{1} x_{2}\right) \in I^{i}$,

$\left(\psi_{1}^{i} \odot \psi_{1}^{i i}\right)\left(\left(x_{2}, y_{2}\right),\left(x_{1}, y_{1}\right)\left(x_{2}, y_{2}\right)\right)=\psi_{1}^{i}\left(x_{2}, x_{1} x_{2}\right) \wedge \mu_{1}^{i i}\left(y_{1}\right) \wedge \mu_{1}^{i i}\left(y_{2}\right), \quad$ if $x_{1} \neq x_{2}, y_{1} \neq y_{2},\left(x_{2}, x_{1} x_{2}\right) \in I^{i}$,

$\left(\psi_{1}^{i} \odot \psi_{1}^{i i}\right)\left(\left(x_{1}, y_{2}\right),\left(x_{1}, y_{2}\right)\left(x_{2}, y_{1}\right)\right)=\psi_{1}^{i}\left(x_{1}, x_{1} x_{2}\right) \wedge \mu_{1}^{i i}\left(y_{1}\right) \wedge \mu_{1}^{i i}\left(y_{2}\right), \quad$ if $x_{1} \neq x_{2}, y_{1} \neq y_{2},\left(x_{1}, x_{1} x_{2}\right) \in I^{i}$,

$\left(\psi_{1}^{i} \odot \psi_{1}^{i i}\right)\left(\left(x_{2}, y_{1}\right),\left(x_{1}, y_{2}\right)\left(x_{2}, y_{1}\right)\right)=\psi_{1}^{i}\left(x_{2}, x_{1} x_{2}\right) \wedge \mu_{1}^{i i}\left(y_{1}\right) \wedge \mu_{1}^{i i}\left(y_{2}\right), \quad$ if $x_{1} \neq x_{2}, y_{1} \neq y_{2},\left(x_{2}, x_{1} x_{2}\right) \in I^{i}$,

$\left(\psi_{2}^{i} \odot \psi_{2}^{i i}\right)\left(\left(x_{1}, y_{1}\right),\left(x_{1}, y_{1}\right)\left(x_{1}, y_{2}\right)\right)=\mu_{2}^{i}\left(x_{1}\right) \vee \psi_{2}^{i i}\left(y_{1}, y_{1} y_{2}\right), \quad$ if $x_{1}=x_{2},\left(y_{1}, y_{1} y_{2}\right) \in I^{i i}$,

$\left(\psi_{2}^{i} \odot \psi_{2}^{i i}\right)\left(\left(x_{1}, y_{2}\right),\left(x_{1}, y_{1}\right)\left(x_{1}, y_{2}\right)\right)=\mu_{2}^{i}\left(m_{1}\right) \vee \psi_{2}^{i i}\left(y_{2}, y_{1} y_{2}\right), \quad$ if $x_{1}=x_{2},\left(y_{2}, y_{1} y_{2}\right) \in I^{i i}$,

$\left(\psi_{2}^{i} \odot \psi_{2}^{i i}\right)\left(\left(x_{1}, y_{1}\right),\left(x_{1}, y_{1}\right)\left(x_{2}, y_{1}\right)\right)=\psi_{2}^{i}\left(x_{1}, x_{1} x_{2}\right) \vee \mu_{2}^{i i}\left(y_{1}\right), \quad$ if $y_{1}=y_{2},\left(x_{1}, x_{1} x_{2}\right) \in I^{i}$,

$\left(\psi_{2}^{i} \odot \psi_{2}^{i i}\right)\left(\left(x_{2}, y_{1}\right),\left(x_{1}, y_{1}\right)\left(x_{2}, y_{1}\right)\right)=\psi_{2}^{i}\left(x_{2}, x_{1} x_{2}\right) \vee \mu_{2}^{i i}\left(y_{1}\right), \quad$ if $y_{1}=y_{2},\left(x_{2}, x_{1} x_{2}\right) \in I^{i}$,

$\left(\psi_{2}^{i} \odot \psi_{2}^{i i}\right)\left(\left(x_{1}, y_{1}\right),\left(x_{1}, y_{1}\right)\left(x_{2}, y_{2}\right)\right)=\psi_{2}^{i}\left(x_{1}, x_{1} x_{2}\right) \vee \mu_{1}^{i i}\left(y_{1}\right) \vee \mu_{1}^{i i}\left(y_{2}\right), \quad$ if $x_{1} \neq x_{2}, y_{1} \neq y_{2},\left(x_{1}, x_{1} x_{2}\right) \in I^{i}$,

$\left(\psi_{2}^{i} \odot \psi_{2}^{i i}\right)\left(\left(x_{2}, y_{2}\right),\left(x_{1}, y_{1}\right)\left(x_{2}, y_{2}\right)\right)=\psi_{2}^{i}\left(x_{2}, x_{1} x_{2}\right) \vee \mu_{1}^{i i}\left(y_{1}\right) \vee \mu_{1}^{i i}\left(y_{2}\right), \quad$ if $x_{1} \neq x_{2}, y_{1} \neq y_{2},\left(x_{2}, x_{1} x_{2}\right) \in I^{i}$,

$\left(\psi_{2}^{i} \odot \psi_{2}^{i i}\right)\left(\left(x_{1}, y_{2}\right),\left(x_{1}, y_{2}\right)\left(x_{2}, y_{1}\right)\right)=\psi_{2}^{i}\left(x_{1}, x_{1} x_{2}\right) \vee \mu_{1}^{i i}\left(y_{1}\right) \wedge \mu_{1}^{i i}\left(y_{2}\right), \quad$ if $x_{1} \neq x_{2}, y_{1} \neq y_{2},\left(x_{1}, x_{1} x_{2}\right) \in I^{i}$,

$\left(\psi_{2}^{i} \odot \psi_{2}^{i i}\right)\left(\left(x_{2}, y_{1}\right),\left(x_{1}, y_{2}\right)\left(x_{2}, y_{1}\right)\right)=\psi_{2}^{i}\left(x_{2}, x_{1} x_{2}\right) \vee \mu_{1}^{i i}\left(y_{1}\right) \wedge \mu_{1}^{i i}\left(y_{2}\right), \quad$ if $x_{1} \neq x_{2}, y_{1} \neq y_{2},\left(x_{2}, x_{1} x_{2}\right) \in I^{i}$. 


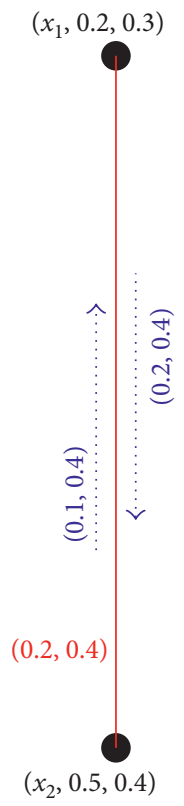

FIGURE 5: $G^{i}$.

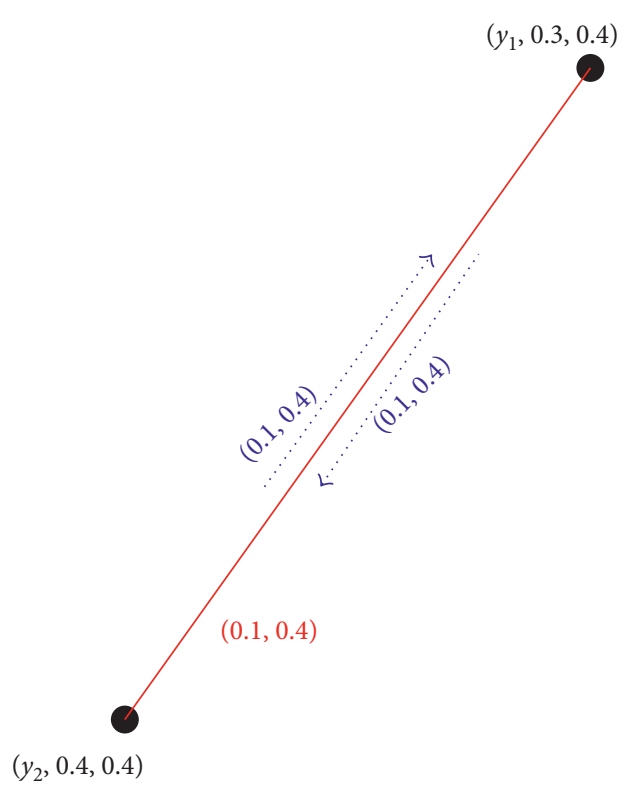

FIgURE 6: $G^{i i}$.

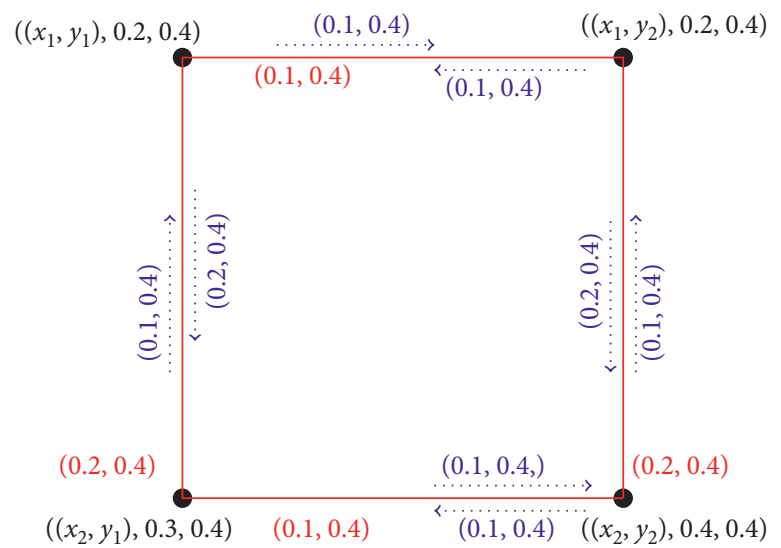

Figure 7: CP of Figures 5 and 6. 
Definition 12. Let $G=G^{i}\left[G^{i i}\right]=\left(V, E, I, \mu^{i} \times \mu^{i i}, v^{i} \times\right.$ $\left.v^{i i}, \psi^{i} \times \psi^{i i}\right)$ be the composition of two IFIGs $G^{i}=\left(V^{i}, E^{i}, I^{i}, \mu^{i}, \nu^{i}, \psi^{i}\right)$ and $G^{i i}=\left(V^{i i}, E^{i i}, I^{i i}, \mu^{i i}, \nu^{i i}, \psi^{i i}\right)$.
Then, the degree of $V=\left(x_{1}, y_{1}\right)$ is represented by $\mathrm{d}_{G^{i}\left[G^{i i}\right]}\left(x_{1}, y_{1}\right)=\left(\mathrm{d}_{1 G^{i}\left[G^{i i}\right]}\left(x_{1}, y_{1}\right), \mathrm{d}_{2 G^{i}\left[G^{i i}\right]}\left(x_{1}, y_{1}\right)\right)$ and defined by

$$
\begin{aligned}
\mathrm{d}_{1 G^{i}\left[G^{i i}\right]}\left(x_{1}, y_{1}\right)= & \sum_{x_{1}=x_{2},\left(y_{1}, y_{1} y_{2}\right) \in I^{i i}} \mu_{1}^{i}\left(x_{1}\right) \vee \psi_{1}^{i i}\left(y_{1}, y_{1} y_{2}\right)+\sum_{y_{1}=y_{2},\left(x_{1}, x_{1} x_{2}\right) \in I^{i}} \psi_{1}^{i}\left(x_{1}, x_{1} x_{2}\right) \wedge \mu_{1}^{i i}\left(y_{1}\right) \\
& +\sum_{y_{1} \neq y_{2},\left(x_{1}, x_{1} x_{2}\right) \in I^{i}} \psi_{1}^{i}\left(x_{1}, x_{1} x_{2}\right) \vee \mu_{1}^{i i}\left(y_{1}\right) \vee \mu_{1}^{i i}\left(y_{2}\right), \\
\mathrm{d}_{2 G^{i}\left[G^{i i}\right]}\left(x_{1}, y_{1}\right)= & \sum_{x_{1}=x_{2},\left(y_{1}, y_{1} y_{2}\right) \in I^{i i}} \mu_{2}^{i}\left(x_{1}\right) \vee \psi_{2}^{i i}\left(y_{1}, y_{1} y_{2}\right)+\sum_{y_{1}=y_{2},\left(x_{1}, x_{1} x_{2}\right) \in I^{i}} \psi_{2}^{i}\left(x_{1}, x_{1} x_{2}\right) \vee \mu_{2}^{i i}\left(l_{1}\right) \\
& +\sum_{y_{1} \neq y_{2},\left(x_{1}, x_{1} x_{2}\right) \in I^{i}} \psi_{1}^{i}\left(x_{1}, x_{1} x_{2}\right) \vee \mu_{1}^{i i}\left(y_{1}\right) \vee \mu_{1}^{i i}\left(y_{2}\right) .
\end{aligned}
$$

Theorem 2. Let $G^{i}=\left(V^{i}, E^{i}, I^{i}, \mu^{i}, \nu^{i}, \psi^{i}\right)$ and $G^{i i}=$ $\left(V^{i i}, E^{i i}, I^{i i}, \mu^{i i}, \nu^{i i}, \psi^{i i}\right)$ be two IFIGs. If $\mu_{1}^{i} \geq \psi_{1}^{i i}, \mu_{2}^{i} \leq \psi_{2}^{i i}$ and $\mu_{1}^{i i} \geq \psi_{1}^{i}, \mu_{2}^{i i} \leq \psi_{2}^{i}$, then $\quad d_{G^{i}\left[G^{i i}\right]}\left(x_{1}, y_{1}\right)=\left|V^{i i}\right| d_{G^{i}}\left(x_{1}\right)$ $+d_{G^{i i}}\left(y_{1}\right)$.
Proof. In the composition of two IFIGs by definition of degree of a vertex, we have

$$
\begin{aligned}
& \mathrm{d}_{1 G^{i}\left[G^{i i}\right]}\left(x_{1}, y_{1}\right)=\sum_{x_{1}=x_{2},\left(y_{1}, y_{1} y_{2}\right) \in I^{i i}} \mu_{1}^{i}\left(x_{1}\right) \wedge \psi_{1}^{i i}\left(y_{1}, y_{1} y_{2}\right)+\sum_{y_{1}=y_{2},\left(x_{1}, x_{1} x_{2}\right) \in I^{i}} \psi_{1}^{i}\left(x_{1}, x_{1} x_{2}\right) \wedge \mu_{1}^{i i}\left(y_{1}\right) \\
& +\sum_{y_{1} \neq y_{2},\left(x_{1}, x_{1} x_{2}\right) \in I^{i}} \psi_{1}^{i}\left(x_{1}, x_{1} x_{2}\right) \wedge \mu_{1}^{i i}\left(y_{1}\right) \wedge \mu_{1}^{i i}\left(y_{2}\right) \text {, } \\
& =\sum_{\left(y_{1}, y_{1} y_{2}\right) \in I^{i i}} \psi_{1}^{i i}\left(y_{1}, y_{1} y_{2}\right)+\sum_{y_{1}=y_{2},\left(x_{1}, x_{1} x_{2}\right) \in I^{i}} \psi_{1}^{i}\left(x_{1}, x_{1} x_{2}\right)+\sum_{y_{1} \neq y_{2},\left(x_{1}, x_{1} x_{2}\right) \in I^{i}} \psi_{1}^{i}\left(x_{1}, x_{1} x_{2}\right) \\
& =\sum_{y_{1}=y_{2},\left(x_{1}, x_{1} x_{2}\right) \in I^{i}} \psi_{1}^{i}\left(x_{1}, x_{1} x_{2}\right)+\sum_{y_{1} \neq y_{2},\left(x_{1}, x_{1} x_{2}\right) \in I^{i}} \psi_{1}^{i}\left(x_{1}, x_{1} x_{2}\right)+\sum_{\left(y_{1}, y_{1} y_{2}\right) \in I^{i i}} \psi_{1}^{i i}\left(y_{1}, y_{1} y_{2}\right) \\
& =\left|V^{i i}\right| \mathrm{d}_{1 G^{i}}\left(x_{1}\right)+\mathrm{d}_{1 G^{i i}}\left(y_{1}\right) \text {. } \\
& \mathrm{d}_{2 G^{i}\left[G^{i i}\right]}\left(x_{1}, y_{1}\right)=\sum_{x_{1}=x_{2},\left(y_{1}, y_{1} y_{2}\right) \in I^{i i}} \mu_{2}^{i}\left(x_{1}\right) \vee \psi_{2}^{i i}\left(y_{1}, y_{1} y_{2}\right)+\sum_{y_{1}=y_{2},\left(x_{1}, x_{1} x_{2}\right) \in I^{i}} \psi_{2}^{i}\left(x_{1}, x_{1} x_{2}\right) \vee \mu_{2}^{i i}\left(y_{1}\right) \\
& +\sum_{y_{1} \neq y_{2},\left(x_{1}, x_{1} x_{2}\right) \in I^{i}} \psi_{2}^{i}\left(x_{1}, x_{1} x_{2}\right) \vee \mu_{1}^{i i}\left(y_{1}\right) \vee \mu_{2}^{i i}\left(y_{2}\right) \text {, } \\
& =\sum_{\left(y_{1}, y_{1} y_{2}\right) \in I^{i i}} \psi_{2}^{i i}\left(y_{1}, y_{1} y_{2}\right)+\sum_{y_{1}=y_{2},\left(x_{1}, x_{1} x_{2}\right) \in I^{i}} \psi_{2}^{i}\left(x_{1}, x_{1} x_{2}\right)+\sum_{y_{1} \neq y_{2},\left(x_{1}, x_{1} x_{2}\right) \in I^{i}} \psi_{2}^{i}\left(x_{1}, x_{1} x_{2}\right) \\
& =\sum_{y_{1}=y_{2},\left(x_{1}, x_{1} x_{2}\right) \in I^{i}} \psi_{2}^{i}\left(x_{1}, x_{1} x_{2}\right)+\sum_{y_{1} \neq y_{2},\left(x_{1}, x_{1} x_{2}\right) \in I^{i}} \psi_{2}^{i}\left(x_{1}, x_{1} x_{2}\right)+\sum_{\left(y_{1}, y_{1} y_{2}\right) \in I^{i i}} \psi_{2}^{i i}\left(y_{1}, y_{1} y_{2}\right) \\
& =\left|V^{i i}\right| \mathrm{d}_{2 G^{i}}\left(x_{1}\right)+\mathrm{d}_{2 G^{i i}}\left(y_{1}\right) \text {. }
\end{aligned}
$$

This implies $\mathrm{d}_{G^{i}\left[G^{i i}\right]}\left(x_{1}, y_{1}\right)=\left|V^{i i}\right| \mathrm{d}_{G^{i}}\left(x_{1}\right)+\mathrm{d}_{G^{i i}}\left(y_{1}\right)$.

Example 6. The composition of graphs given in Figures 5 and 6 is shown in Figure 8. Here, $\mu_{1}^{i} \geq \psi_{1}^{i i}, \mu_{2}^{i} \leq \psi_{2}^{i i}$ and $\mu_{1}^{i i} \geq \psi_{1}^{i}, \mu_{2}^{i i} \leq \psi_{2}^{i}$; then, by Theorem 2, $\mathrm{d}_{1 G^{i}\left[G^{i i}\right]}\left(x_{1}, y_{1}\right)$
$=\left|V^{i i}\right| \mathrm{d}_{1 G^{i}} \quad\left(x_{1}\right)+\mathrm{d}_{1 G^{i i}}\left(y_{1}\right)=2 \times 0.2+0.1=0.5, \mathrm{~d}_{2 G^{i}\left[G^{i i}\right]}$ $\left(x_{1}, y_{1}\right)=\left|V^{i i}\right| \mathrm{d}_{2 G^{i}}\left(x_{1}\right)+\mathrm{d}_{2 G^{i i}}\left(y_{1}\right)=2 \times 0.4+0.4=1.2$. So, $\mathrm{d}_{G^{i}\left[G^{i i}\right]}\left(x_{1}, y_{1}\right)=(0.5,1.2)$. In the same way, we can examine the degree of the remaining vertices given in Figure 8. 


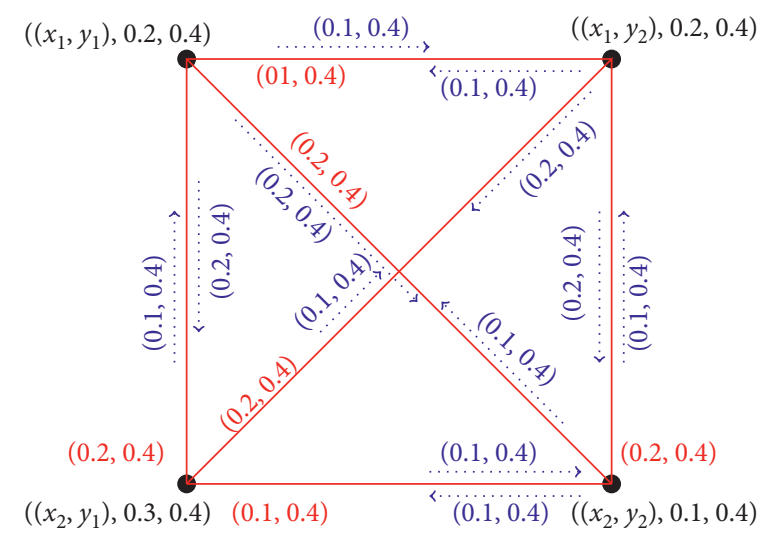

FIgURE 8: Composition of IFIGs given in Figures 5 and 6.

\section{Degree of a Vertex of TP and NP of Intuitionistic Fuzzy Incidence Graphs}

This section explained the idea of the degree of a vertex in TP and NP of two IFIGs.
Definition 13. The TP of two IFIGs $G^{i}=\left(V^{i}, E^{i}, I^{i}, \mu^{i}, \nu^{i}, \psi^{i}\right)$ and $G^{i i}=\left(V^{i i}, E^{i i}, I^{i i}, \mu^{i i}, \nu^{i i}, \psi^{i i}\right)$ is defined as an IFIG, $G=G^{i} \diamond G^{i i}=\left(V, E, I, \mu^{i} \diamond \mu^{i i}, \nu^{i} \diamond \nu^{i i}, \psi^{i} \diamond \psi^{i i}\right)$, where $V=$ $V^{i} \times V^{i i}, \quad E=\left\{\left(\left(x_{1}, y_{1}\right),\left(x_{2}, y_{2}\right)\right) \mid\left(x_{1}, x_{2}\right) \in E^{i}, \quad\left(y_{1}, y_{2}\right) \in\right.$ $E^{i i}$, and $I=\left\{\left(x_{1}, y_{1}\right),\left(x_{1}, y_{1}\right)\left(x_{1}, y_{2}\right) \mid\left(x_{1}, x_{1} x_{2}\right) \in I^{i}\right.$, $\left(x_{2}, x_{1} x_{2}\right) \in I^{i},\left(y_{1}, y_{1} y_{2}\right) \in I^{i i},\left(y_{2}, y_{1} y_{2}\right) \in I^{i i}$ with

$$
\begin{aligned}
& \left(\mu_{1}^{i} \diamond \mu_{1}^{i i}\right)\left(x_{1}, y_{1}\right)=\mu_{1}^{i}\left(x_{1}\right) \wedge \mu_{1}^{i i}\left(y_{1}\right), \quad \forall\left(x_{1}, y_{1}\right) \in V^{i} \diamond V^{i i}, \\
& \left(\mu_{2}^{i} \diamond \mu_{2}^{i i}\right)\left(x_{1}, y_{1}\right)=\mu_{2}^{i}\left(x_{1}\right) \vee \mu_{2}^{i i}\left(y_{1}\right), \quad \forall\left(x_{1}, y_{1}\right) \in V^{i} \diamond V^{i i}, \\
& \left(v_{1}^{i} \diamond v_{1}^{i i}\right)\left(\left(x_{1}, y_{1}\right),\left(x_{2}, y_{2}\right)\right)=v_{1}^{i}\left(x_{1}, x_{2}\right) \wedge v_{1}^{i i}\left(y_{1}, y_{2}\right) \forall\left(x_{1}, x_{2}\right) \in E^{i}, \quad\left(y_{1}, y_{2}\right) \in E^{i i}, \\
& \left(v_{2}^{i} \diamond v_{2}^{i i}\right)\left(\left(x_{1}, y_{1}\right),\left(x_{2}, y_{2}\right)\right)=v_{2}^{i}\left(x_{1}, x_{2}\right) \vee v_{2}^{i i}\left(y_{1}, y_{2}\right) \forall\left(x_{1}, x_{2}\right) \in E^{i}, \quad\left(y_{1}, y_{2}\right) \in E^{i i}, \\
& \left(\psi_{1}^{i} \diamond \psi_{1}^{i i}\right)\left(\left(x_{1}, y_{1}\right),\left(x_{1}, y_{1}\right)\left(x_{2}, y_{2}\right)\right)=\psi_{1}^{i}\left(x_{1}, x_{1} x_{2}\right) \wedge \psi_{1}^{i i}\left(y_{1}, y_{1} y_{2}\right) \forall\left(x_{1}, x_{1} x_{2}\right) \in I^{i},\left(y_{1}, y_{1} y_{2}\right) \in I^{i i} \text {, } \\
& \left(\psi_{1}^{i} \diamond \psi_{1}^{i i}\right)\left(\left(x_{2}, y_{2}\right),\left(x_{1}, y_{1}\right)\left(x_{2}, y_{2}\right)\right)=\psi_{1}^{i}\left(x_{2}, x_{1} x_{2}\right) \wedge \psi_{1}^{i i}\left(y_{2}, y_{1} y_{2}\right) \forall\left(x_{2}, x_{1} x_{2}\right) \in I^{i},\left(y_{2}, y_{1} y_{2}\right) \in I^{i i} \text {, } \\
& \left(\psi_{1}^{i} \diamond \psi_{1}^{i i}\right)\left(\left(x_{1}, y_{2}\right),\left(x_{1}, y_{2}\right)\left(x_{2}, y_{1}\right)\right)=\psi_{1}^{i}\left(x_{1}, x_{1} x_{2}\right) \wedge \psi_{1}^{i i}\left(y_{2}, y_{1} y_{2}\right) \forall\left(x_{1}, x_{1} x_{2}\right) \in I^{i}, \quad\left(y_{2}, y_{1} y_{2}\right) \in I^{i i}, \\
& \left(\psi_{1}^{i} \diamond \psi_{1}^{i i}\right)\left(\left(x_{2}, y_{1}\right),\left(x_{1}, y_{2}\right)\left(x_{2}, y_{1}\right)\right)=\psi_{1}^{i}\left(x_{2}, x_{1} x_{2}\right) \wedge \psi_{1}^{i i}\left(y_{1}, y_{1} y_{2}\right) \forall\left(x_{2}, x_{1} x_{2}\right) \in I^{i}, \quad\left(y_{1}, y_{1} y_{2}\right) \in I^{i i}, \\
& \left(\psi_{2}^{i} \diamond \psi_{2}^{i i}\right)\left(\left(x_{1}, y_{1}\right),\left(x_{1}, y_{1}\right)\left(x_{2}, y_{2}\right)\right)=\psi_{2}^{i}\left(x_{1}, x_{1} x_{2}\right) \vee \psi_{2}^{i i}\left(y_{1}, y_{1} y_{2}\right) \forall\left(x_{1}, x_{1} x_{2}\right) \in I^{i}, \quad\left(y_{1}, y_{1} y_{2}\right) \in I^{i i}, \\
& \left(\psi_{2}^{i} \diamond \psi_{2}^{i i}\right)\left(\left(x_{2}, y_{2}\right),\left(x_{1}, y_{1}\right)\left(x_{2}, y_{2}\right)\right)=\psi_{2}^{i}\left(x_{2}, x_{1} x_{2}\right) \vee \psi_{2}^{i i}\left(y_{2}, y_{1} y_{2}\right) \forall\left(x_{2}, x_{1} x_{2}\right) \in I^{i}, \quad\left(y_{2}, y_{1} y_{2}\right) \in I^{i i}, \\
& \left(\psi_{2}^{i} \diamond \psi_{2}^{i i}\right)\left(\left(x_{1}, y_{2}\right),\left(x_{1}, y_{2}\right)\left(x_{2}, y_{1}\right)\right)=\psi_{2}^{i}\left(x_{1}, x_{1} x_{2}\right) \vee \psi_{2}^{i i}\left(y_{2}, y_{1} y_{2}\right) \forall\left(x_{1}, x_{1} x_{2}\right) \in I^{i}, \quad\left(y_{2}, y_{1} y_{2}\right) \in I^{i i}, \\
& \left(\psi_{2}^{i} \diamond \psi_{2}^{i i}\right)\left(\left(x_{2}, y_{1}\right),\left(x_{1}, y_{2}\right)\left(x_{2}, y_{1}\right)\right)=\psi_{2}^{i}\left(x_{2}, x_{1} x_{2}\right) \vee \psi_{2}^{i i}\left(y_{1}, y_{1} y_{2}\right) \forall\left(x_{2}, x_{1} x_{2}\right) \in I^{i}, \quad\left(y_{1}, y_{1} y_{2}\right) \in I^{i i} .
\end{aligned}
$$


Definition 14. Let $G=G^{i} \diamond G^{i i}=\left(V, E, I, \mu^{i} \times \mu^{i i}, \nu^{i} \times v^{i i}\right.$, $\left.\psi^{i} \times \psi^{i i}\right)$ be the TP of two IFIGs $G^{i}=\left(V^{i}, E^{i}, I^{i}, \mu^{i}, v^{i}, \psi^{i}\right)$ and $G^{i i}=\left(V^{i i}, E^{i i}, I^{i i}, \mu^{i i}, \nu^{i i}, \psi^{i i}\right)$. Then, the degree of
$V=\left(x_{1}, y_{1}\right) \quad$ is shown by $\mathrm{d}_{G^{i} \diamond G^{i i}}\left(x_{1}, y_{1}\right)=$ $\left(\mathrm{d}_{1 G^{i} \diamond G^{i i}}\left(x_{1}, y_{1}\right), \mathrm{d}_{2 G^{i} \diamond G^{i i}}\left(x_{1}, y_{1}\right)\right)$ and defined by

$$
\begin{aligned}
& \mathrm{d}_{1 G^{i} \diamond G^{i i}}\left(x_{1}, y_{1}\right)=\sum_{\left(x_{1}, x_{1} x_{2}\right) \in I^{i},\left(y_{1}, y_{1} y_{2}\right) \in I^{i i}} \psi_{1}^{i}\left(x_{1}, x_{1} x_{2}\right) \wedge \psi_{1}^{i i}\left(y_{1}, y_{1} y_{2}\right), \\
& \mathrm{d}_{2 G^{i} \diamond G^{i i}}\left(x_{1}, y_{1}\right)=\sum_{\left(x_{1}, x_{1} x_{2}\right) \in I^{i},\left(y_{1}, y_{1} y_{2}\right) \in I^{i i}} \psi_{2}^{i}\left(x_{1}, x_{1} x_{2}\right) \vee \psi_{2}^{i i}\left(y_{1}, y_{1} y_{2}\right) .
\end{aligned}
$$

Theorem 3. Let $G^{i}=\left(V^{i}, E^{i}, I^{i}, \mu^{i}, \nu^{i}, \psi^{i}\right)$ and $G^{i i}=\left(V^{i i}\right.$, $\left.E^{i i}, I^{i i}, \mu^{i i}, \nu^{i i}, \psi^{i i}\right)$ be two IFIGs. If $\psi_{1}^{i i} \geq \psi_{1}^{i}, \psi_{2}^{i i} \leq \psi_{2}^{i}$, then Proof. Suppose $\psi_{1}^{i i} \geq \psi_{1}^{i}, \psi_{2}^{i i} \leq \psi_{2}^{i}$; then, $d_{G^{i} \diamond G^{i i}}\left(x_{1}, y_{1}\right)=d_{G^{i}}\left(x_{1}\right)$ and if $\psi_{1}^{i} \geq \psi_{1}^{i i}, \psi_{2}^{i} \leq \psi_{2}^{i i}$, then $d_{G^{i} \diamond G^{i i}}\left(x_{1}, y_{1}\right)=d_{G^{i i}}\left(y_{1}\right)$.

$$
\begin{aligned}
\mathrm{d}_{1 G^{i} \diamond G^{i i}}\left(x_{1}, y_{1}\right) & =\sum_{\left(\left(x_{1}, x_{1} y_{1}\right),\left(x_{2}, x_{2} y_{2}\right)\right) \in I}\left(\psi_{1}^{i} \diamond \psi_{1}^{i i}\right) \\
\left(\left(x_{1}, y_{1}\right)\left(x_{2}, y_{2}\right)\right) & =\sum_{\left(x_{1}, x_{1} x_{2}\right) \in I^{i},\left(y_{1}, y_{1} y_{2}\right) \in I^{i i}} \psi_{1}^{i}\left(x_{1}, x_{1} x_{2}\right) \wedge \psi_{1}^{i i}\left(y_{1}, y_{1} y_{2}\right) \\
& =\sum \psi_{1}^{i}\left(x_{1}, x_{1} x_{2}\right)=\mathrm{d}_{1 G^{i}}\left(x_{1}\right), \\
\mathrm{d}_{2 G^{i} \diamond G^{i i}}\left(x_{1}, y_{1}\right) & =\sum_{\left(x_{1}, x_{1} x_{2}\right) \in I^{i},\left(y_{1}, y_{1} y_{2}\right) \in I^{i i}} \psi_{1}^{i}\left(x_{1}, x_{1} x_{2}\right) \vee \psi_{2}^{i i}\left(y_{1}, y_{1} y_{2}\right)=\sum \psi_{2}^{i}\left(x_{1}, x_{1} x_{2}\right)=\mathrm{d}_{2 G^{i}}\left(x_{1}\right) .
\end{aligned}
$$

This implies $\mathrm{d}_{G^{i} \diamond G^{i i}}\left(x_{1}, y_{1}\right)=\mathrm{d}_{G^{i}}\left(x_{1}\right)$. In a similar way, if $\psi_{1}^{i} \geq \psi_{1}^{i i}, \psi_{2}^{i} \leq \psi_{2}^{i i}$, then $\mathrm{d}_{G^{i} \diamond G^{i i}}\left(x_{1}, y_{1}\right)=\mathrm{d}_{G^{i i}}\left(y_{1}\right)$.

Example 7. Let $G^{i}$ and $G^{i i}$ be two IFIGs as shown in Figures 9 and 10, and their TP is provided in Figure 11 with $\psi_{1}^{i i} \geq \psi_{1}^{i}, \psi_{2}^{i i} \leq \psi_{2}^{i}$; then, by Theorem $3, \mathrm{~d}_{1 G^{i} \diamond G^{i i}}\left(x_{1}, y_{1}\right)=0.1=$ $\mathrm{d}_{1 G^{i}}\left(x_{1}\right)$ and $\mathrm{d}_{2 G^{i} \diamond G^{i i}}\left(x_{1}, y_{1}\right)=0.4=\mathrm{d}_{2 G^{i}}\left(x_{1}\right)$. Hence, $\mathrm{d}_{G^{i} \diamond G^{i i}}\left(x_{1}, y_{1}\right)=(0.1,0.4)$.

Finally, we discussed the way to explore the degree of a vertex in NP of two IFIGs.
Definition 15. The NP of two IFIGs $G^{i}=\left(V^{i}, E^{i}, I^{i}, \mu^{i}, \nu^{i}, \psi^{i}\right)$ and $G^{i i}=\left(V^{i i}, E^{i i}, I^{i i}, \mu^{i i}, \nu^{i i}, \psi^{i i}\right)$ is defined as an IFIG $G=$ $G^{i} \oplus G^{i i}=\left(V, E, I, \mu^{i} \oplus \mu^{i i}, \nu^{i} \oplus \nu^{i i}, \psi^{i} \oplus \psi^{i i}\right)$ where $V=V^{i} \times V^{i i}$, $E=\left\{\left(\left(x_{1}, y_{1}\right),\left(x_{2}, y_{2}\right)\right) \mid x_{1}=x_{2},\left(y_{1}, y_{2}\right) \in E^{i i}\right.$ or $y_{1}=y_{2}$, $\left(x_{1}, x_{2}\right) \in E^{i} \cup\left\{\left(\left(x_{1}, y_{1}\right),\left(x_{2}, y_{2}\right)\right) \mid\left(x_{1}, x_{2}\right) \in E^{i},\left(y_{1}, y_{2}\right) \in\right.$ $\left.E^{i i}\right\}$, and $I=\left\{\left(x_{1}, y_{1}\right),\left(x_{1}, y_{1}\right)\left(x_{1}, y_{2}\right) \mid x_{1}=x_{2},\left(y_{1}, y_{1} y_{2}\right)\right.$ $\in I^{i i},\left(y_{2}, y_{1} y_{2}\right) \in I^{i i}$ or $y_{1}=y_{2},\left(x_{1}, x_{1} x_{2}\right) \in I^{i},\left(x_{2}, x_{1} x_{2}\right)$ $\in I^{i} \cup\left\{\left(x_{1}, y_{1}\right),\left(x_{2}, y_{2}\right) \mid\left(x_{1}, x_{1} x_{2}\right) \in I^{i},\left(x_{2}, x_{1} x_{2}\right) \in I^{i}\right.$, $\left.\left(y_{1}, y_{1} y_{2}\right) \in I^{i i},\left(y_{2}, y_{1} y_{2}\right) \in I^{i i}\right\}$ with 


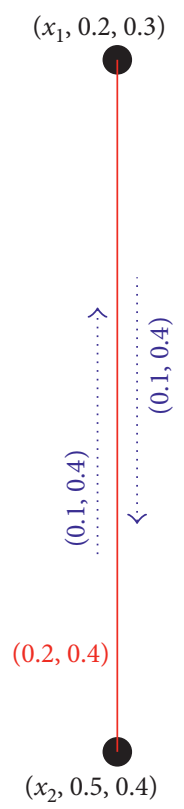

Figure 9: $G^{i}$.

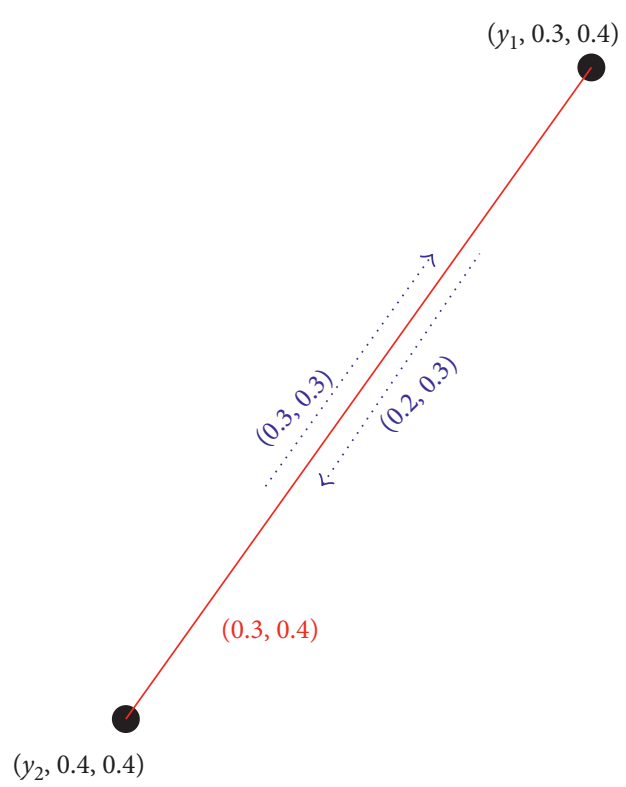

FIgURe 10: $G^{i i}$.

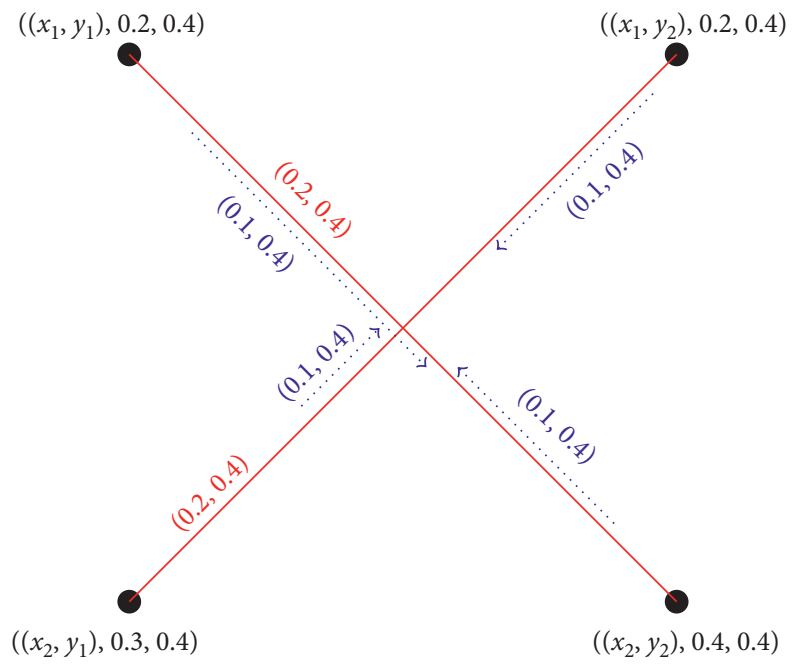

FIGURe 11: TP of IFIGs given in Figures 9 and 10. 


$$
\begin{aligned}
& \left(\mu_{1}^{i} \oplus \mu_{1}^{i i}\right)\left(x_{1}, y_{1}\right)=\mu_{1}^{i}\left(x_{1}\right) \wedge \mu_{1}^{i i}\left(y_{1}\right), \quad \forall\left(x_{1}, y_{1}\right) \in V^{i} \oplus V^{i i}, \\
& \left(\mu_{2}^{i} \oplus \mu_{2}^{i i}\right)\left(x_{1}, y_{1}\right)=\mu_{2}^{i}\left(x_{1}\right) \vee \mu_{2}^{i i}\left(y_{1}\right), \quad \forall\left(x_{1}, y_{1}\right) \in V^{i} \oplus V^{i i}, \\
& \left(v_{1}^{i} \oplus v_{1}^{i i}\right)\left(\left(x_{1}, y_{1}\right)\left(x_{2}, y_{2}\right)\right)= \begin{cases}\mu_{1}^{i}\left(x_{1}\right) \wedge v_{1}^{i i}\left(y_{1}, y_{2}\right), & \text { if } x_{1}=x_{2},\left(y_{1}, y_{2}\right) \in E^{i i}, \\
v_{1}^{i}\left(x_{1}, x_{2}\right) \wedge \mu_{1}^{i i}\left(y_{1}\right), & \text { if } y_{1}=y_{2},\left(x_{1}, x_{2}\right) \in E^{i}, \\
v_{1}^{i}\left(x_{1}, x_{2}\right) \wedge v_{1}^{i i}\left(y_{1}, y_{2}\right), & \text { if } x_{1} \neq x_{2}, y_{1} \neq y_{2},\left(x_{1}, x_{2}\right) \in E^{i} \text { and }\left(y_{1}, y_{2}\right) \in E^{i i},\end{cases} \\
& \left(v_{2}^{i} \oplus \nu_{2}^{i i}\right)\left(\left(x_{1}, y_{1}\right)\left(x_{2}, y_{2}\right)\right)= \begin{cases}\mu_{2}^{i}\left(x_{1}\right) \vee v_{2}^{i i}\left(y_{1}, y_{2}\right), & \text { if } x_{1}=x_{2},\left(y_{1}, y_{2}\right) \in E^{i i}, \\
v_{2}^{i}\left(x_{1}, x_{2}\right) \vee \mu_{2}^{i i}\left(y_{1}\right), & \text { if } y_{1}=y_{2},\left(x_{1}, x_{2}\right) \in E^{i}, \\
v_{2}^{i}\left(x_{1}, x_{2}\right) \vee v_{2}^{i i}\left(y_{1}, y_{2}\right), & \text { if } x_{1} \neq x_{2}, y_{1} \neq y_{2},\left(x_{1}, x_{2}\right) \in E^{i} \text { and }\left(y_{1}, y_{2}\right) \in E^{i i},\end{cases}
\end{aligned}
$$

$\left(\psi_{1}^{i} \oplus \psi_{1}^{i i}\right)\left(\left(x_{1}, y_{1}\right),\left(x_{1}, y_{1}\right)\left(x_{1}, y_{2}\right)\right)=\mu_{1}^{i}\left(x_{1}\right) \wedge \psi_{1}^{i i}\left(y_{1}, y_{1} y_{2}\right), \quad$ if $x_{1}=x_{2},\left(y_{1}, y_{1} y_{2}\right) \in I^{i i}$,

$\left(\psi_{1}^{i} \oplus \psi_{1}^{i i}\right)\left(\left(x_{1}, y_{2}\right),\left(x_{1}, y_{1}\right)\left(x_{1}, y_{2}\right)\right)=\mu_{1}^{i}\left(x_{1}\right) \wedge \psi_{1}^{i i}\left(y_{2}, y_{1} y_{2}\right), \quad$ if $x_{1}=x_{2},\left(y_{2}, y_{1} y_{2}\right) \in I^{i i}$,

$\left(\psi_{1}^{i} \oplus \psi_{1}^{i i}\right)\left(\left(x_{1}, y_{1}\right),\left(x_{1}, y_{1}\right)\left(x_{2}, y_{1}\right)\right)=\psi_{1}^{i}\left(x_{1}, x_{1} x_{2}\right) \wedge \mu_{1}^{i i}\left(y_{1}\right), \quad$ if $y_{1}=y_{2},\left(x_{1}, x_{1} x_{2}\right) \in I^{i}$,

$\left(\psi_{1}^{i} \oplus \psi_{1}^{i i}\right)\left(\left(x_{2}, y_{1}\right),\left(x_{1}, y_{1}\right)\left(x_{2}, y_{1}\right)\right)=\psi_{1}^{i}\left(x_{2}, x_{1} x_{2}\right) \wedge \mu_{1}^{i i}\left(y_{1}\right), \quad$ if $y_{1}=y_{2},\left(x_{2}, x_{1} x_{2}\right) \in I^{i}$,

$\left(\psi_{1}^{i} \oplus \psi_{1}^{i i}\right)\left(\left(x_{1}, y_{1}\right),\left(x_{1}, y_{1}\right)\left(x_{2}, y_{2}\right)\right)=\psi_{1}^{i}\left(x_{1}, x_{1} x_{2}\right) \wedge \psi_{1}^{i i}\left(y_{1}, y_{1} y_{2}\right), \quad$ if $x_{1} \neq x_{2}, y_{1} \neq y_{2}$,

$$
\left(x_{1}, x_{1} x_{2}\right) \in I^{i},\left(y_{1}, y_{1} y_{2}\right) \in I^{i i},
$$

$\left(\psi_{1}^{i} \oplus \psi_{1}^{i i}\right)\left(\left(x_{2}, y_{2}\right),\left(x_{1}, y_{1}\right)\left(x_{2}, y_{2}\right)\right)=\psi_{1}^{i}\left(x_{2}, x_{1} x_{2}\right) \wedge \psi_{1}^{i i}\left(y_{2}, y_{1} y_{2}\right), \quad$ if $x_{1} \neq x_{2}, y_{1} \neq y_{2}$,

$$
\left(x_{2}, x_{1} x_{2}\right) \in I^{i},\left(y_{2}, y_{1} y_{2}\right) \in I^{i i} \text {, }
$$

$\left(\psi_{1}^{i} \oplus \psi_{1}^{i i}\right)\left(\left(x_{1}, y_{2}\right),\left(x_{1}, y_{2}\right)\left(x_{2}, y_{1}\right)\right)=\psi_{1}^{i}\left(x_{1}, x_{1} x_{2}\right) \wedge \psi_{1}^{i i}\left(y_{2}, y_{1} y_{2}\right), \quad$ if $x_{1} \neq x_{2}, y_{1} \neq y_{2}$,

$$
\left(x_{1}, x_{1} x_{2}\right) \in I^{i},\left(y_{2}, y_{1} y_{2}\right) \in I^{i i},
$$

$\left(\psi_{1}^{i} \oplus \psi_{1}^{i i}\right)\left(\left(x_{2}, y_{1}\right),\left(x_{1}, y_{2}\right)\left(x_{2}, y_{1}\right)\right)=\psi_{1}^{i}\left(x_{2}, x_{1} x_{2}\right) \wedge \psi_{1}^{i i}\left(y_{1}, y_{1} y_{2}\right), \quad$ if $x_{1} \neq x_{2}, y_{1} \neq y_{2}$,

$$
\left(x_{2}, x_{1} x_{2}\right) \in I^{i},\left(y_{1}, y_{1} y_{2}\right) \in I^{i i},
$$

$\left(\psi_{2}^{i} \oplus \psi_{2}^{i i}\right)\left(\left(x_{1}, y_{1}\right),\left(x_{1}, y_{1}\right)\left(x_{1}, y_{2}\right)\right)=\mu_{1}^{i}\left(x_{1}\right) \vee \psi_{2}^{i i}\left(y_{1}, y_{1} y_{2}\right), \quad$ if $x_{1}=x_{2},\left(y_{1}, y_{1} y_{2}\right) \in I^{i i}$,

$\left(\psi_{2}^{i} \oplus \psi_{2}^{i i}\right)\left(\left(x_{1}, y_{2}\right),\left(x_{1}, y_{1}\right)\left(x_{1}, y_{2}\right)\right)=\mu_{1}^{i}\left(x_{1}\right) \vee \psi_{2}^{i i}\left(y_{2}, y_{1} y_{2}\right), \quad$ if $x_{1}=x_{2},\left(y_{2}, y_{1} y_{2}\right) \in I^{i i}$,

$\left(\psi_{2}^{i} \oplus \psi_{2}^{i i}\right)\left(\left(x_{1}, y_{1}\right),\left(x_{1}, y_{1}\right)\left(x_{2}, y_{1}\right)\right)=\psi_{2}^{i}\left(x_{1}, x_{1} x_{2}\right) \vee \mu_{1}^{i i}\left(y_{1}\right), \quad$ if $y_{1}=y_{2},\left(x_{1}, x_{1} x_{2}\right) \in I^{i}$,

$\left(\psi_{2}^{i} \oplus \psi_{2}^{i i}\right)\left(\left(x_{2}, y_{1}\right),\left(x_{1}, y_{1}\right)\left(x_{2}, y_{1}\right)\right)=\psi_{2}^{i}\left(x_{2}, x_{1} x_{2}\right) \vee \mu_{1}^{i i}\left(y_{1}\right), \quad$ if $y_{1}=y_{2},\left(x_{2}, x_{1} x_{2}\right) \in I^{i}$,

$\left(\psi_{2}^{i} \oplus \psi_{2}^{i i}\right)\left(\left(x_{1}, y_{1}\right),\left(x_{1}, y_{1}\right)\left(x_{2}, y_{2}\right)\right)=\psi_{2}^{i}\left(x_{1}, x_{1} x_{2}\right) \vee \psi_{2}^{i i}\left(y_{1}, y_{1} y_{2}\right), \quad$ if $x_{1} \neq x_{2}, y_{1} \neq y_{2}$,

$$
\left(x_{1}, x_{1} x_{2}\right) \in I^{i},\left(y_{1}, y_{1} y_{2}\right) \in I^{i i},
$$

$\left(\psi_{2}^{i} \oplus \psi_{2}^{i i}\right)\left(\left(x_{2}, y_{2}\right),\left(x_{1}, y_{1}\right)\left(x_{2}, y_{2}\right)\right)=\psi_{2}^{i}\left(x_{2}, x_{1} x_{2}\right) \vee \psi_{2}^{i i}\left(y_{2}, y_{1} y_{2}\right), \quad$ if $x_{1} \neq x_{2}, y_{1} \neq y_{2}$,

$$
\left(x_{2}, x_{1} x_{2}\right) \in I^{i},\left(y_{2}, y_{1} y_{2}\right) \in I^{i i} \text {, }
$$

$\left(\psi_{2}^{i} \oplus \psi_{2}^{i i}\right)\left(\left(x_{1}, y_{2}\right),\left(x_{1}, y_{2}\right)\left(x_{2}, y_{1}\right)\right)=\psi_{2}^{i}\left(x_{1}, x_{1} x_{2}\right) \vee \psi_{2}^{i i}\left(y_{2}, y_{1} y_{2}\right), \quad$ if $x_{1} \neq x_{2}, y_{1} \neq y_{2}$,

$$
\left(x_{1}, x_{1} x_{2}\right) \in I^{i},\left(y_{2}, y_{1} y_{2}\right) \in I^{i i},
$$

$\left(\psi_{2}^{i} \oplus \psi_{2}^{i i}\right)\left(\left(x_{2}, y_{1}\right),\left(x_{1}, y_{2}\right)\left(x_{2}, y_{1}\right)\right)=\psi_{2}^{i}\left(x_{2}, x_{1} x_{2}\right) \vee \psi_{2}^{i i}\left(y_{1}, y_{1} y_{2}\right), \quad$ if $x_{1} \neq x_{2}, y_{1} \neq y_{2}$,

$$
\left(x_{2}, x_{1} x_{2}\right) \in I^{i},\left(y_{1}, y_{1} y_{2}\right) \in I^{i i} \text {. }
$$


Definition 16. Let $G=G^{i} \oplus G^{i i}=\left(V, E, I, \mu^{i} \oplus \mu^{i i}, \nu^{i} \oplus \nu^{i i}\right.$, $\left.\psi^{i} \oplus \psi^{i i}\right)$ be the NP of two IFIGs $G^{i}=\left(V^{i}, E^{i}, I^{i}, \mu^{i}, v^{i}, \psi^{i}\right)$ and $G^{i i}=\left(V^{i i}, E^{i i}, I^{i i}, \mu^{i i}, \nu^{i i}, \psi^{i i}\right)$. Then, the degree of
$V=\left(x_{1}, y_{1}\right) \quad$ is shown by $\mathrm{d}_{G^{i} \oplus G^{i i}}\left(x_{1}, y_{1}\right)=$ $\left(\mathrm{d}_{1 G^{i} \oplus G^{i i}}\left(x_{1}, y_{1}\right), \mathrm{d}_{2 G^{i} \oplus G^{i i}}\left(x_{1}, y_{1}\right)\right)$ and defined by

$$
\begin{aligned}
\mathrm{d}_{1 G^{i} \oplus G^{i i}}\left(x_{1}, y_{1}\right)= & \sum_{x_{1}=x_{2},\left(y_{1}, y_{1} y_{2}\right) \in I^{i i}} \mu_{1}^{i}\left(x_{1}\right) \wedge \psi_{1}^{i i}\left(y_{1}, y_{1} y_{2}\right)+\sum_{y_{1}=y_{2},\left(x_{1}, x_{1} x_{2}\right) \in I^{i}} \psi_{1}^{i}\left(x_{1}, x_{1} x_{2}\right) \wedge \mu_{1}^{i i}\left(y_{1}\right) \\
& +\sum_{\left(x_{1}, x_{1} x_{2}\right) \in I^{i},\left(y_{1}, y_{1} y_{2}\right) \in I^{i i}} \psi_{1}^{i}\left(x_{1}, x_{1} x_{2}\right) \wedge \psi_{1}^{i i}\left(y_{1}, y_{1} y_{2}\right), \\
\mathrm{d}_{2 G^{i} \oplus G^{i i}}\left(x_{1}, y_{1}\right)= & \sum_{x_{1}=x_{2},\left(y_{1}, y_{1} y_{2}\right) \in I^{i i}} \mu_{2}^{i}\left(x_{1}\right) \vee \psi_{2}^{i i}\left(y_{1}, y_{1} y_{2}\right)+\sum_{y_{1}=y_{2},\left(x_{1}, x_{1} x_{2}\right) \in I^{i}} \psi_{2}^{i}\left(x_{1}, x_{1} x_{2}\right) \vee \mu_{1}^{i i}\left(y_{1}\right) \\
& +\sum_{\left(x_{1}, x_{1} x_{2}\right) \in I^{i},\left(y_{1}, y_{1} y_{2}\right) \in I^{i i}} \psi_{2}^{i}\left(x_{1}, x_{1} x_{2}\right) \vee \psi_{2}^{i i}\left(y_{1}, y_{1} y_{2}\right) .
\end{aligned}
$$

Theorem 4. Let $G^{i}=\left(V^{i}, E^{i}, I^{i}, \mu^{i}, \nu^{i}, \psi^{i}\right)$ and $G^{i i}=\left(V^{i i}, E^{i i}\right.$, $\left.I^{i i}, \mu^{i i}, \nu^{i i}, \psi^{i i}\right)$ be two IFIGs. If $\mu_{1}^{i} \geq \psi_{1}^{i i}$, $\mu_{2}^{i} \leq \psi_{2}^{i i}, \mu_{1}^{i i} \geq \psi_{1}^{i}, \mu_{2}^{i i} \leq \psi_{2}^{i}, \psi_{1}^{i} \leq \psi_{1}^{i i}$ and $\psi_{2}^{i} \geq \psi_{2}^{i i}$, then $d_{G^{i} \oplus G^{i i}}\left(x_{1}, y_{1}\right)=\left|V^{i i}\right| d_{G^{i}}\left(x_{1}\right)+d_{G^{i i}}\left(y_{1}\right)$.
Proof. Let $\mu_{1}^{i} \geq \psi_{1}^{i i}, \mu_{2}^{i} \leq \psi_{2}^{i i}, \mu_{1}^{i i} \geq \psi_{1}^{i}, \mu_{2}^{i i} \leq \psi_{2}^{i}, \psi_{1}^{i} \leq \psi_{1}^{i i}$, and $\psi_{2}^{i} \geq \psi_{2}^{i i}$; then, we have

$$
\begin{aligned}
\mathrm{d}_{1 G^{i} \oplus G^{i i}}\left(x_{1}, y_{1}\right)= & \sum_{x_{1}=x_{2},\left(y_{1}, y_{1} y_{2}\right) \in I^{i i}} \mu_{1}^{i}\left(x_{1}\right) \wedge \psi_{1}^{i i}\left(y_{1}, y_{1} y_{2}\right)+\sum_{y_{1}=y_{2},\left(x_{1}, x_{1} x_{2}\right) \in I^{i}} \psi_{1}^{i}\left(x_{1}, x_{1} x_{2}\right) \wedge \mu_{1}^{i i}\left(y_{1}\right) \\
& +\sum_{\left(x_{1}, x_{1} x_{2}\right) \in I^{i},\left(y_{1}, y_{1} y_{2}\right) \in I^{i i}}^{i}\left(x_{1}, x_{1} x_{2}\right) \wedge \psi_{1}^{i i}\left(y_{1}, y_{1} y_{2}\right), \\
= & \sum_{\left(y_{1}, y_{1} y_{2}\right) \in I^{i i}} \psi_{1}^{i i}\left(y_{1}, y_{1} y_{2}\right)+\sum_{y_{1}=y_{2},\left(x_{1}, x_{1} x_{2}\right) \in I^{i}} \psi_{1}^{i}\left(x_{1}, x_{1} x_{2}\right)+\sum_{\left(x_{1}, x_{1} x_{2}\right) \in I^{i}} \psi_{1}^{i}\left(x_{1}, x_{1} x_{2}\right) \\
= & \sum_{y_{1}=y_{2},\left(x_{1}, x_{1} x_{2}\right) \in I^{i}} \psi_{1}^{i}\left(x_{1}, x_{1} x_{2}\right)+\sum_{\left(x_{1}, x_{1} x_{2}\right) \in I^{i}} \psi_{1}^{i}\left(x_{1}, x_{1} x_{2}\right)+\sum_{\left(y_{1}, y_{1} y_{2}\right) \in I^{i i}} \psi_{1}^{i i}\left(y_{1}, y_{1} y_{2}\right) \\
= & \left|V^{i i}\right| \mathrm{d}_{1 G^{i}}\left(k_{1}\right)+\mathrm{d}_{1 G^{i i}}\left(l_{1}\right), \\
\mathrm{d}_{2 G^{i} \oplus G^{i i}}\left(x_{1}, y_{1}\right)= & \sum_{x_{1}=x_{2},\left(y_{1}, y_{1} y_{2}\right) \in I^{i i}} \mu_{2}^{i}\left(x_{1}\right) \vee \psi_{2}^{i i}\left(y_{1}, y_{1} y_{2}\right)+\sum_{y_{1}=y_{2},\left(x_{1}, x_{1} x_{2}\right) \in I^{i}} \psi_{2}^{i}\left(x_{1}, x_{1} x_{2}\right) \vee \mu_{2}^{i i}\left(y_{1}\right) \\
& +\sum_{\left(x_{1}, x_{1} x_{2}\right) \in I^{i},\left(y_{1}, y_{1} y_{2}\right) \in I^{i i}}^{i}\left(x_{1}, x_{1} x_{2}\right) \vee \psi_{2}^{i i}\left(y_{1}, y_{1} y_{2}\right), \\
= & \sum_{\left(y_{1}, y_{1} y_{2}\right) \in I^{i i}} \psi_{1}^{i i}\left(y_{1}, y_{1} y_{2}\right)+\sum_{y_{1}=y_{2},\left(x_{1}, x_{1} x_{2}\right) \in I^{i}} \psi_{2}^{i}\left(x_{1}, x_{1} x_{2}\right)+\sum_{\left(x_{1}, x_{1} x_{2}\right) \in I^{i}} \psi_{2}^{i}\left(x_{1}, x_{1} x_{2}\right) \\
= & \sum_{y_{1}=y_{2},\left(x_{1}, x_{1} x_{2}\right) \in I^{i}} \psi_{2}^{i}\left(x_{1}, x_{1} x_{2}\right)+\sum_{\left(x_{1}, x_{1} x_{2}\right) \in I^{i}} \psi_{2}^{i}\left(x_{1}, x_{1} x_{2}\right)+\sum_{\left(y_{1}, y_{1} y_{2}\right) \in I^{i i}} \psi_{1}^{i i}\left(y_{1}, y_{1} y_{2}\right) \\
= & \left|V^{i i}\right| \mathrm{d}_{2 G^{i}}\left(x_{1}\right)+\mathrm{d}_{1 G^{i i}}\left(y_{1}\right) .
\end{aligned}
$$

This implies $\mathrm{d}_{G^{i} \oplus G^{i i}}\left(x_{1}, y_{1}\right)=\left|V^{i i}\right| \mathrm{d}_{G^{i}}\left(x_{1}\right)+\mathrm{d}_{G^{i i}}\left(y_{1}\right)$.

Example 8. Let $G^{i}$ and $G^{i i}$ be two IFIGs as shown in Figures 12 and 13 and their NP is given in Figure 14 with $\mu_{1}^{i} \geq \psi_{1}^{i i}, \mu_{2}^{i} \leq \psi_{2}^{i i}, \mu_{1}^{i i} \geq \psi_{1}^{i}, \mu_{2}^{i i} \leq \psi_{2}^{i}, \psi_{1}^{i} \leq \psi_{1}^{i i}$, and $\psi_{2}^{i} \geq \psi_{2}^{i i}$; then, by Theorem 4, $\mathrm{d}_{1 G^{i} \oplus G^{i i}}\left(x_{1}, y_{1}\right)=0.6=\mathrm{d}_{1 G^{i}}\left(x_{1}\right)$ and $\mathrm{d}_{2 G^{i} \oplus G^{i i}}\left(x_{1}, y_{1}\right)=0.9=\mathrm{d}_{2 G^{i}}\left(y_{1}\right)$. Hence, $\mathrm{d}_{G^{i} \oplus G^{i i}}\left(x_{1}, y_{1}\right)$ $=(0.6,0.9)$.

\section{Real-Life Application of $\mathrm{CP}$ and Composition of IFIGs}

Here, we include an application of $\mathrm{CP}$ and the composition of two IFIGs. As an illustrative case, consider two networks (IFIGs) $G^{i}$ and $G^{i i}$ with respective two and two vertices showing different departments of different branches of a textile industry. The MS value of the vertices shows the 


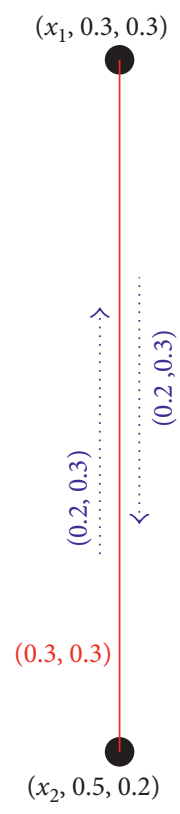

FIGURE 12: $G^{i}$.

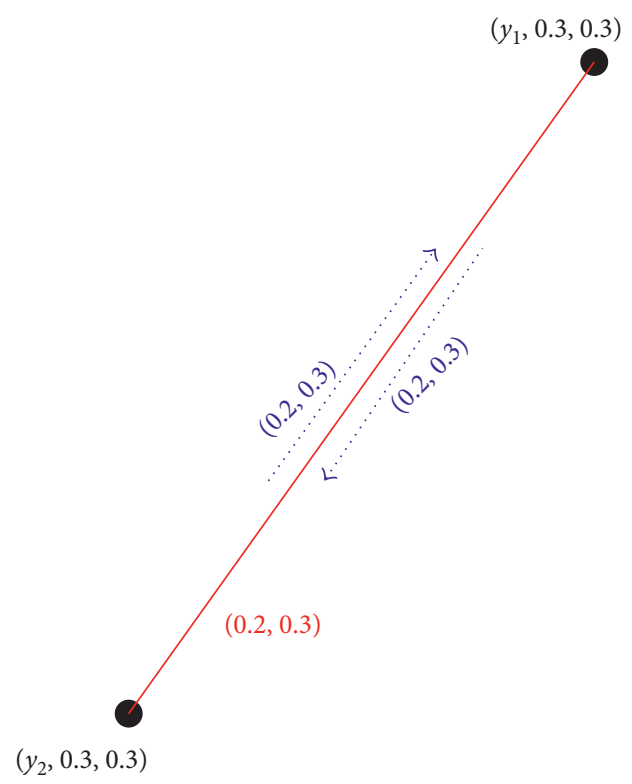

Figure 13: $G^{i i}$.

percentage of the working staff in a department and the NMS value of the vertices represents the nonworking staff. The MS value of the edges shows the mutual collaboration of the departments with each other and the NMS value of the edges shows the noncollaboration of the departments with one another. The MS value of IPs shows the percentage of progress and NMS value shows the percentage of nonprogress of the departments. As in Figure 7 and Figure 8, CP and composition of $G^{i}$ and $G^{i i}$ show the percentage of progress of department $x_{1}$ with departments $y_{1}$ and $y_{2}$ has the maximum MS value and the percentage of nonprogress of department $x_{1}$ with departments $y_{1}$ and $y_{2}$ has the lesser

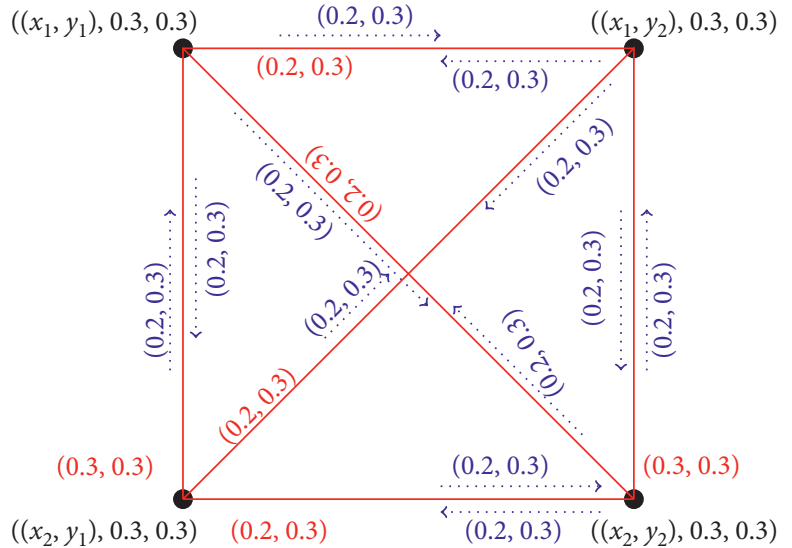

Figure 14: NP of IFIGs provided in Figures 12 and 13.

NMS value. Therefore, these are the most appropriate combinations of departments showing the maximum percentage of progress and a minimum percentage of nonprogress of the textile industry among all departments.

\section{Comparative Analysis}

We have applied the model on CP of two IFIGs provided in Figure 7 and we get the following results. In Figure 7, there are two possible paths from vertex $\left(x_{1}, y_{1}\right)$ to vertex $\left(x_{1}, y_{2}\right)$ such as $P_{1}:\left(x_{1}, y_{1}\right),\left(\left(x_{1}, y_{1}\right),\left(x_{1}, y_{1}\right)\left(x_{1}, y_{2}\right)\right),\left(\left(x_{1}, y_{1}\right)\right.$ $\left.\left(x_{1}, y_{2}\right)\right),\left(\left(x_{1}, y_{2}\right),\left(x_{1}, y_{1}\right)\left(x_{1}, y_{2}\right)\right),\left(x_{1}, y_{2}\right)$ and $P_{2}:\left(x_{1}\right.$, $\left.y_{1}\right), \quad\left(\left(x_{1}, y_{1}\right),\left(x_{1}, y_{1}\right)\left(x_{2}, y_{1}\right)\right),\left(\left(x_{1}, y_{1}\right)\left(x_{2}, y_{1}\right)\right), \quad\left(\left(x_{2}\right.\right.$, $\left.\left.y_{1}\right),\left(x_{1}, y_{1}\right)\left(x_{2}, y_{1}\right)\right),\left(x_{1}, y_{2}\right)$. The incidence strength (IS) of $P_{1}$ is the minimum value of all MS values of all the IPs of $P_{1}$ and the maximum value of all NMS values of all the IPs of $P_{1}$. Therefore, the IS $\left(P_{1}\right)=\wedge\{0.1,0.2\}=0.1, \vee\{0.4,0.4\}=0.4=$ $(0.1,0.4)$ and $I S\left(P_{2}\right)=(0.1,0.4)$. The greatest $\left(I S^{\infty}\right)$ is the maximum value of MS values of IS $\left(P_{1}\right)$ and IS $\left(P_{2}\right)$ and the minimum values of NMS values of IS $\left(P_{1}\right)$ and IS $\left(P_{2}\right)$. This implies $I S^{\infty}\left(\left(x_{1}, y_{1}\right),\left(x_{1}, y_{2}\right)\right)=(0.1,0.4)$. Similarly, the $I S^{\infty}\left(\left(x_{1}, y_{1}\right),\left(x_{2}, y_{1}\right)\right)=(0.1,0.4), \quad I S^{\infty}\left(\left(x_{1}, y_{2}\right),\left(x_{2}\right.\right.$, $\left.\left.y_{2}\right)\right)=(0.1,0.4)$, and $I S^{\infty}\left(\left(x_{2}, y_{1}\right),\left(x_{2}, y_{2}\right)\right)=(0.1,0.4)$. Here, all the $I S^{\infty}$ are the same but, in our model, the degrees of all the vertices of CP of two IFIGs $\mathrm{d}_{G^{i} \times G^{i i}}$ $\left(x_{1}, y_{1}\right)=(0.3,0.8)=\mathrm{d}_{G^{i} \times G^{i i}}\left(x_{1}, y_{2}\right)$ and $\mathrm{d}_{G^{i} \times G^{i i}}\left(x_{2}, y_{1}\right)$ $=(0.2,0.8)=\mathrm{d}_{G^{i} \times G^{i i}}\left(x_{2}, y_{2}\right)$ are not all same.

By applying the model on the composition of two IFIGs given in Figure 8 , we get $I S^{\infty}\left(\left(x_{1}, y_{1}\right), \quad\left(x_{1}\right.\right.$, $\left.\left.y_{2}\right)\right)=(0.1,0.4), \quad I S^{\infty}\left(\left(x_{1}, y_{1}\right),\left(x_{2}, y_{1}\right)\right)=(0.1,0.4), \quad I S^{\infty}$ $\left(\left(x_{1}, y_{2}\right),\left(x_{2}, y_{2}\right)\right)=(0.1,0.4)$, and $I S^{\infty}\left(\left(x_{2}, y_{1}\right),\left(x_{2}, y_{2}\right)\right)$ $=(0.1,0.4)$. In our model, the degrees of the vertices of the composition of two IFIGs $\mathrm{d}_{G^{i} \odot G^{i i}}\left(x_{1}, y_{1}\right)=(0.5,1.2)$ $=\mathrm{d}_{G^{i} \odot G^{i i}}\left(x_{1}, y_{2}\right)$ and $\mathrm{d}_{G^{i} \odot G^{i i}}\left(x_{2}, y_{1}\right)=(0.3,1.2)=\mathrm{d}_{G^{i} \odot G^{i i}}$ $\left(x_{2}, y_{2}\right)$ are not all the same. It can be observed that the degree of the vertices $\mathrm{d}_{G^{i} \odot G^{i i}}\left(x_{1}, y_{1}\right)=(0.5,1.2)=\mathrm{d}_{G^{i} \odot G^{i i}}$ $\left(x_{1}, y_{2}\right)$ shows the percentage of progress of department $x_{1}$ with departments $y_{1}$ and $y_{2}$ has the maximum MS value and the percentage of nonprogress of departments $y_{1}$ and $y_{2}$ has the lesser NMS value. Due to this reason, the existing model is not helpful to decide which departments have the percentage of progress that has the maximum 
MS value and the percentage of nonprogress that has the minimum value. The method to find IS and $I S^{\infty}$ of a single network of all possible paths between vertex $x_{i}$ and $y_{i}$ is explained in the existing model but there is no clue to find IS and $I S^{\infty}$ of the product of networks. The existing model is beneficial for single networks but not suitable to explain the overall impact of products of different networks. But in our model, we can discuss the overall impact of the combination of different networks with the help of CP, composition, TP, and NP. Our model deals with not only a single network but also more than one network. In this way, we can talk about the overall impact of the products of different networks. Therefore, our proposed method is better than the existing method.

\section{Conclusion}

This study makes explicit the several structures of IFIGs and their properties. Further research can be carried out to explore the structure of IFIGs which would have applications in different fields. In this research article, we examined the degree of the vertices of the IFIGs $G^{i} \times G^{i i}, G^{i}\left[G^{i i}\right], G^{i} \diamond G^{i i}$, and $G^{i} \oplus G^{i i}$ under certain terms and conditions and demonstrated them with various examples. We also established some new results on the degree of a vertex in terms of theorems. The degree of any graph plays a key role in studying the foundational characteristic of a graph in detail. This study is beneficial to explore different kinds of properties of IFIGs such as $G^{i} \times G^{i i}, G^{i}\left[G^{i i}\right], G^{i} \diamond G^{i i}$, and $G^{i} \oplus G^{i i}$. An application of CP and composition of IFIGs in the textile industry is also provided. Our study will be instrumental to apprehend the further attributes of IFIGs at length. The main benefit of our study is that it will be supportive to find the percentage of progress that has maximum MS value and percentage of nonprogress that has minimum NMS value in different universities, garment factories, and hospitals. Another advantage of our study is that we do not have any need to calculate the $I S$ and $I S^{\infty}$ of all possible paths from vertex $u_{i}$ to vertex $v_{i}$ to find the neutrosophic incidence cut-pair. We have to just calculate the degree of the vertices to find the percentage of progress having maximum MS value and percentage of nonprogress having minimum NMS value of different departments. The vertex having maximum MS value and minimum NMS value will be the required vertex. Therefore, our proposed study is better than the existing neutrosophic IGs. The authors propose to extend further these concepts to other types of IFIGs such as soft IFIGs, competition IFIGs, interval-valued IFIGs, threshold IFIGs, Hamiltonian IFIGs, the coloring of IFIGs, and bipolar IFIGs.

\section{Data Availability}

No data were used to support this study.

\section{Conflicts of Interest}

The authors declare that they have no conflicts of interest.

\section{References}

[1] L. A. Zadeh, "Fuzzy sets," Information and Control, vol. 8, no. 3, pp. 338-353, 1965.

[2] K. T. Atanassov, Intuitionistic Fuzzy sets: Theory and Applications, pp. 1-137, Springer, New York, NY, USA, 1999.

[3] S. K. De, R. Biswas, and A. R. Roy, "An application of intuitionistic fuzzy sets in medical diagnosis," Fuzzy Sets and Systems, vol. 117, no. 2, pp. 209-213, 2001.

[4] $\mathrm{Z}$. $\mathrm{Xu}$, "Intuitionistic fuzzy aggregation operators," IEEE Transactions on Fuzzy Systems, vol. 15, no. 6, pp. 1179-1187, 2007.

[5] A. Rosenfeld, "Fuzzy sets and their applications to cognitive and decision processes," in Proceedings of the U.S.-Japan Seminar on Fuzzy Sets and Their Applications, pp. 77-95, Berkeley, CA, USA, July 1974.

[6] J. N. Mordeson and P. Chang-Shyh, "Operations on fuzzy graphs," Information Sciences, vol. 79, no. 3-4, pp. 159-170, 1994.

[7] M. Pal, S. Samanta, and H. Rashmanlou, "Some results on interval-valued fuzzy graphs," International Journal of Computer Science and Electronic Engineering, vol. 3, no. 3, pp. 205-211, 2015.

[8] H. Rashmanlou and M. Pal, "Some properties of highly irregular interval-valued fuzzy graphs," World Applied Sciences Journal, vol. 27, no. 12, pp. 1756-1773, 2013.

[9] N. Jan, K. Ullah, T. Mahmood et al., "Some root level modifications in interval valued fuzzy graphs and their generalizations including neutrosophic graphs," Mathematics, vol. 7, no. 1, p. 72, 2019.

[10] B. Mathew, S. J. John, and H. Garg, "Vertex rough graphs," Complex and Intelligent Systems, vol. 6, no. 3, pp. 1-7, 2020.

[11] S. Samanta and M. Pal, "Fuzzy threshold graphs," CIIT International Journal of Fuzzy Systems, vol. 3, no. 12, pp. 360364, 2011.

[12] S. Samanta and M. Pal, "Fuzzy tolerance graphs," International Journal of Latest Trends in Mathematics, vol. 1, no. 2, pp. 57-67, 2011.

[13] S. Samanta and M. Pal, "Bipolar fuzzy hypergraphs," International Journal of Fuzzy Logic Systems, vol. 2, no. 1, pp. 17-28, 2012.

[14] G. Ghorai and M. Pal, "On some operations and density of $m$ polar fuzzy graphs," Pacific Science Review A: Natural Science and Engineering, vol. 17, no. 1, pp. 14-22, 2015.

[15] G. Ghorai and M. Pal, "Certain types of product bipolar fuzzy graphs," International Journal of Applied and Computational Mathematics, vol. 3, no. 2, pp. 605-619, 2017.

[16] S. Goyal, P. Garg, and V. N. Mishra, "New composition of graphs and their wiener indices," Applied Mathematics and Nonlinear Sciences, vol. 4, no. 1, pp. 175-180, 2019.

[17] R. Parvathi and M. Karunambigai, "Intuitionistic fuzzy graphs," Computational Intelligence, Theory and Applications, pp. 139-150, Springer, New York, NY, USA, 2006.

[18] R. Parvathi, M. G. Karunambigai, and K. T. Atanassov, "Operations on intuitionistic fuzzy graphs," in Proceedings of the FUZZ-IEEE 2009, IEEE International Conference on Fuzzy Systems, Jeju Island, Korea, August 2009.

[19] A. N. Gani and S. S. Begum, "Degree, order and size in intuitionistic fuzzy graphs," International Journal of Algorithms, Computing and Mathematics, vol. 3, no. 3, pp. 11-16, 2010.

[20] S. Sahoo and M. Pal, "Different types of products on intuitionistic fuzzy graphs," Pacific Science Review A: Natural Science and Engineering, vol. 17, no. 3, pp. 87-96, 2015. 
[21] S. Sahoo and M. Pal, "Intuitionistic fuzzy competition graphs," Journal of Applied Mathematics and Computing, vol. 52, no. 1-2, pp. 37-57, 2016.

[22] S. Sahoo and M. Pal, "Intuitionistic fuzzy tolerance graphs with application," Journal of Applied Mathematics and Computing, vol. 55, no. 1-2, pp. 495-511, 2017.

[23] S. Sahoo and M. Pal, "Product of intuitionistic fuzzy graphs and degree," Journal of Intelligent \& Fuzzy Systems, vol. 32, no. 1, pp. 1059-1067, 2017.

[24] S. Sahoo, S. Kosari, H. Rashmanlou, and M. Shoib, "New concepts in intuitionistic fuzzy graph with application in water supplier systems," Mathematics, vol. 8, no. 8, p. 1241, 2020.

[25] A. Bozhenyuk, S. Belyakov, M. Knyazeva, and I. Rozenberg, "On computing domination set in intuitionistic fuzzy graph," International Journal of Computational Intelligence Systems, vol. 14, no. 1, pp. 617-624, 2021.

[26] T. Dinesh, "Fuzzy incidence graph-an introduction," $A d$ vances in Fuzzy Sets and Systems, vol. 21, no. 1, pp. 33-48, 2016.

[27] D. S. Malik, S. Mathew, and J. N. Mordeson, "Fuzzy incidence graphs," Fuzzy Graph Theory with Applications to Human Trafficking, pp. 87-137, Springer, New York, NY, USA, 2018.

[28] D. S. Malik, S. Mathew, and J. N. Mordeson, "Fuzzy incidence graphs: applications to human trafficking," Information Sciences, vol. 447, pp. 244-255, 2018.

[29] S. Mathew and J. N. Mordeson, "Connectivity concepts in fuzzy incidence graphs," Information Sciences, vol. 382-383, pp. 326-333, 2017.

[30] J. Fang, I. Nazeer, T. Rashid, and J.-B. Liu, "Connectivity and Wiener index of fuzzy incidence graphs," Mathematical Problems in Engineering, vol. 2021, Article ID 6682966, 7 pages, 2021. 